\title{
A suitable conditioning regimen in allogeneic hematopoietic stem cell transplantation for adult patients with acute lymphoblastic leukemia
}

\author{
Masahiro Imamura*
}

\begin{abstract}
Adult acute lymphoblastic leukemia (ALL) is one of the intractable diseases in hematological malignancies, showing about $30 \%$ long-term survival by intensive chemotherapies with high incidence of relapse. Allogeneic hematopoietic stem cell transplantation (allogeneic HSCT) has been therefore applied to many such patients, showing better responses than chemotherapy and autologous HSCT, although there were some controversial results regarding a timing of allogeneic HSCT, especially in standard-risk ALL patients. In general, a better outcome (about $40 \%$ to $60 \%$ long-term survival) is obtained when adult high-risk patients in first complete remission (CR1) or second complete remission (CR2) received allogeneic HSCT using conventional myeloablative conditioning (MAC) regimens.Long-termsurvival decreased depending on the remission status; namely, about $20-40 \%$ in CR2, $10-20 \%$ in $\geqq \mathrm{CR} 3$, and about $10 \%$ in non-CR. The most common MAC regimens for allogeneic HSCT in ALL patients are $120 \mathrm{mg} / \mathrm{kg}$ cyclophosphamide (CY) plus 12 to 13.2 gray (Gy) fractionated total body irradiation (TBI). By these conditioning regimens, the 3-year overall survival (OS)

CR1.In fact, when we analyzedthedata for 515 patients aged 15 to 59 years who received allogeneic HSCT in CR1 or CR2 with CY/TBI regimen between 1993 and 2007 from the Japan Society for Hematopoietic Cell Transplantation data base and the Japan Marrow Donor Program data base, the 5-year OS rate was $55.2 \%$. In contrast, we have used medium-dose VP-16 in addition to conventional $\mathrm{CY} / \mathrm{TBI}$ regimen to eradicate minimal residual disease. This regimen showed an excellent outcome in adult ALL patients transplanted in CR1, resulting in the 3-year OS rate of $89.2 \%$ without increasing the 3 -year relapse rate $(8.1 \%)$ and transplant-related mortality (TRM) rate (5.4\%). Reduced-intensity conditioning (RIC) regimens are also applied for adult ALL patients with favorable outcomes (the 2- to 3- year OS rate: $30 \%$ to $60 \%$ ); however, the 2- to 3year relapse rate $(20 \%$ to $40 \%)$ and TRM rate $(20 \%$ to $30 \%)$ are still high. Therefore, a suitable conditioning regimen in allogeneic HSCT for adult ALL patients aged younger 50 years old in CR1 appears to be medium-dose $\mathrm{VP} / \mathrm{CY} / \mathrm{TBI}$, while RIC regimens are preferable for patients aged over 50 years old or younger patients with some complications.
\end{abstract} rate of about $50 \%$ was obtained when it was performed in 


\title{
HaploidenticalFamilyDonor for Hematopoietic Transplantation toTreat High Risk Hematological Diseases in Adult
}

\author{
Ming Yao, Shang-Ju Wu, Jih-Luh Tang,Yao-Chang Chen*
}

\begin{abstract}
Haploidenticalfamily donor is an alternative for whom indicated for allogeneic hematopoietic stem cell transplantation (allo-HSCT). Beneficially that is readily available in comparison to matched unrelated donor (MUD) searching. We describe our experience with allo-HSCT in 21 adult patients (age: 16-61 y/o median 26) with high-risk hematological diseases. Among them, 18 patients (86\%) with acute leukemia, 1 lymphoma and 2 severe aplastic anemia (SAA). Nineteen (90\%) were parental donors and 2 filial donors. Fourteen (67\%) were HLA-DR identical. Hematopoietic stem cells were mobilized by G-CSF and collected peripherally, which was not manipulated in vitro. Among 19 patients with hematological malignancies, 15 (79\%) received myeloablative conditioning and 4 reduced intensity conditioning, but they all received ATG containingimmunoablation therapy before allo-HSCT. Post-transplantationalGvHD prophylaxis consisted of cyclosporine and MTX/MMF. All patients had successful trilineage engraftment. G-CSF was routinely given after transplantation. However, delayed graft failure developed in 2 SAA patients and they were successfully rescued by

second allo-HSCT with the same donor. Three (14\%) patients had Grade III/IV acute GvHD, while3 in 11 (27\%) evaluable patients suffered from chronic GvHD. All patients hadcytomegalovirus (CMV) hyperantigenemia and 2 developed CMV peumonitis. Epstein-Barr virus (EBV) viremia was found in 10 patients, with onset time from Day 18 to Day 272. Among them, 4 patients developed post transplantation lymphoproliferative disorder (PTLD). Fourteen (67\%) patients died post allo-HSCT. The cause of death included $6(43 \%)$ relapse, $4(29 \%)$ infection, 2 (14\%) PTLD, 1 (7\%) GVHD and 1 (7\%) poor graft function. The overall survival (OS) for 19 patients with hematological malignancies at 1 and $2 \mathrm{yr}$ was $49 \%$ and $42 \%$ respectively. That is similar in comparison with 62 high risk leukemia patients who received MUD allo-HSCT during the same period at the same BMT unit, their OS at 1 and $2 \mathrm{yr}$ was $46 \%$ and $44 \%$ respectively.In conclusion, our results suggest that haploidenticalfamily donors are feasible alternatives for allo-HSCT in patients withhigh-risk hematological diseases under an intensive immunoablation conditioning regimen.
\end{abstract}




\title{
Proceedings of the 2 nd Meeting of the Asian Cellular Therapy Organization
}

\section{Hematopoietic stem cell transplantation in China}

\author{
Kaiyan LIU*
}

\begin{abstract}
Hematopoietic stem cell transplantation (HSCT) has been applied in China for about 30 years, the number of HSCT has been increasing, donor and stem cell sources were expanded, indication of diseases and patients for HSCT extended.Up to now, 102 medical units nationwide have earned the certificate to perform HSCT. The annual increase rates were $8.8 \%$ to $10.8 \%$. Matched sibling donor is $41 \%$, mismatched related/haploidentical donor is $24 \%$, unrelated volunteer donor is $16 \%$, and umbilical cord blood is $2 \%$. The indications of major disease entities are acute myeloid leukemia (AML, 35\%), acute lymphobastic leukemia (ALL, 25\%), chronic myeloid leukemia (CML, 21\%), and myelodysplastic syndrome (MDS, 8\%).The most encouraging outcome of related haploidentical HSCT in the world has been proved in the novel system without ex vivo T-depletion due to the contributions that Chinese doctors made.In contrast to

CD34+ selected related haplotype transplants, Peking University developed an unmanipulatedhaploidentical transplantation with granulocyte colony-stimulating factor (G-CSF) primed marrow grafts and G-CSF mobilized peripheral blood grafts (G-PB) as stem cell source. Comparable incidence of graft-versus-host disease (GVHD), transplantation- related mortality (TRM) and the probability of survival were found among patients who received haplotype transplants and those from HLA identical sibling or unrelated HSCT. Consequently, the total number of related haploidentical HSCT is increasing during the past four years, and now accounts for about 30\% of that of whole allogeneic HSCT.The Chinese Stem Cell Donor Database Management Center was established in 2001. As of August 31, 2010, there are 1149189 volunteers in the Chinese Marrow Donor Program (CMDP), with 1807 blood stem cell cases.
\end{abstract}




\title{
Depletion of Alloreactive T cells for Tolerance Induction in a Recipient of Kidney and Hematopoietic Stem Cell Transplantations
}

\author{
Kanjana Tangnararatchakit ${ }^{1}$, Wiwat Teerapanich ${ }^{2}$, Usanarat Anurathapan ${ }^{1}$, Wiwat Tapaneeyaolarn ${ }^{1}$, Samart \\ Pakakasama $^{1}$, Saengsuree Jootar ${ }^{3}$, Shimon Slavin ${ }^{4}$, Suradej Hongeng ${ }^{1^{*}}$
}

\begin{abstract}
The present case report represents a successful attempt to induce transplantation tolerance to organ allograft by combined administration of donor hematopoietic cells and kidney based on in vivo deletion of alloreactive host-vsgraft and graft-vs-host alloreactive $T$ cells following nonmyeloablative conditioning. We were able to induce mixed and eventually full donor chimerism and tolerance of kidney allograft in a 15-year-old male with ESRD after cisplatin treatment and autologous HSCT for mediastinal germ cell tumor. Our approach to induce tolerance was based on preferential depletion of alloreactive T cells
\end{abstract}

Abstract induced by exposure to donor's alloantigens and administration of cyclophosphamide at day 2 \& day 3 after stem cells infusion. Additional non-specific immunosuppression as part of the conditioning included exposure to 2 fractions of TLI, treatment with alemtuzumab (monoclonal anti-CD52) and short-term conventional IS treatment to avoid early graft loss, due to request of IRB. Using this approach, with rapid tapering of all conventional IS treatment the patient maintains good renal functions without evidence of both acute and chronic rejection for 25 months off all medications. 


\title{
Current Status of Hematopoietic Stem Cell Transplantation (HSCT) in India
}

\author{
Banavali S
}

\begin{abstract}
Though the first Indian HSCT was done at Tata Memorial Hospital way back in 1983, even today HSCT programme in India is in its infancy. In the first 28 years only 4015 transplants have been done. In USA (population 307 million), 16,974 HSCTs were done in 2008. In comparison, in India with a population of 1.2 billion, 438 transplants were done in 2008 . Presently, there are approximately 25 centers undertaking HSCT in India.
\end{abstract}

Abstract

As opposed to majority of transplants done worldwide being autologous, $60 \%$ of transplants done in India are allogeneic. The main indications for allogeneic transplants were Thallasemia Major 26\% \& acute leukemias 33\%; and for autologous transplants were multiple myeloma $50 \%$ \& lymphomas $31 \%$. Most of the allogeneic HSCTs in India are now done with PBSC as source of stem cells. However, as compared to developed countries, still most donors in India are HLA matched siblings. Very few haplo, MUD or cord blood transplants have been done. Major transplant centers like CMC, Vellore \& TMH, Mumbai have started venturing in this field. However, the extremely high cost of such transplant is still a major source of hindrance.

Though we are improving by leaps and bounds, one of the major reasons for slow progress in the field of HSCT in
India is the limited number of Tertiary Cancer Centers and shortage of trained personnel, both medical and paramedical, in this field. Cost is also a major issue. Though the cost of transplant in India is around USD \$20,000 which is a fraction of the cost for such a transplant in USA, this is still beyond the reach of the majority of patientsin India, which has a per capita GDP of $\$ 3608$ (as opposed \$ 48,665 of USA \& 34,646 of Japan). With no medical insurance system, most patients have to pay from their pockets.

Most of the SCTs done in India are done as part of routine "service". Except at CMC Vellore, hardly any "research" is being undertaken. This also reflects in the fact that a pub med search for "HSCT \& India" yielded only 111 publications. Also most published work is in the preliminary stage and it may take some time before the translational research reaches to the bedside.HSCT is by no means an ideal mode of therapy. What we need in the future is better way to decrease GVHD without affecting the GVL effect. More use of simpler cellular therapies like dendritic cells, mesenchymal stem cells, NK cells, immunomodulators etc. to achieve this and improve outcome of malignant conditions, and hopefully limit the use of HSCT only for non-malignant conditions! 


\title{
Proceedings of the 2 nd Meeting of the Asian Cellular Therapy Organization
}

JSRM Code: 007030700028

\section{Introduction of WHO-WBMT Vietnam Workshop 2011}

\author{
Yoshihisa Kodera ${ }^{*}$
}

\begin{abstract}
Worldwide Network for Blood and Marrow Transplantation (WBMT) is planning the first global level workshop for the promotion of hematopoietic stem cell transplantation (HSCT) in developing countries under the collaboration with World Health Organization (WHO) in Hanoi, Vietnam, on Nov. 10-12, 2011. The preparatory meetings for this Vietnam Workshop 2011 were held in Phuket, Orland, Hawaii and Paris under the attendance of the representatives from Vietnam, WBMT (APBMT/EBMT/CIBMTR/ WMDA etc) and WHO. The goals of the workshop were discussed and were set as followings; 1) Create awareness among government policymakers about the value of hematopoietic stem cell (HSC) transplantation in developing healthcare systems, 2) Encourage the integration of HSC transplantation within the Healthcare Policy of developing countries, 3) Establish the basic ethical, medical and infrastructure requirements for

providing HSC transplantation within a developing healthcare system, 4) Create a model for achieving goals 1) - 3) that can be replicated throughout the WHO regions of the world, 5) Optimize existing transplant programs. Through almost bi-weekly telephone conference, the actual constructions of the workshop have been finalized and those are the followings; 1) Establishing a Transplant Program, 2) Indication for Transplant and Patient Selection, 3) Donor Selection, 4) Graft Processing, 5) Developing an Outcomes Database, 6) Dissemination of Information, 7) Inspection and accreditation( AHCTA). At this moment, 60 attendees from foreign countries (guests from developing countries and speakers from experienced countries) and 100 domestic (Vietnam) attendees are expected. This trial is experimental but we expect that it would be a fruitful one not only for developing countries but also for advanced countries in the field of HSCT.
\end{abstract}




\title{
Autologous hematopoietic stem cell transplantation in the treatment of severe autoimmune disease
}

\author{
Hiroshi Tsukamoto*
}

\begin{abstract}
Some of patients with autoimmune disease (AD), havingprogressive skin sclerosis or interstitial pneumonia (IP) etc., are severely damaged or fatal from the treatment-resistant disease progression.To induce remission in such severe $A D$ by eradicationof autoreactive lymphocytes andby immune reconstitution, we underwent a phase I-II trial to elucidate feasibility and efficacy of autologous hematopoietic stem cell transplantation (auto-HSCT) for severe AD. Nineteen patients with systemic sclerosis (SSc), 3 patients with dermatomyositis and a patient with Wegener's granulomatosis (WG) were enrolled. Peripheral blood stem cells (PBSCs) were mobilized with cyclophosphamide (CY, $4 \mathrm{~g} / \mathrm{m}^{2}$ ) and G-CSF. After collecting PBSCs more than $2 \times 10^{\wedge} 6 \mathrm{CD} 34+$ cells $/ \mathrm{kg}$ by apheresis, they were cryopreserved until autographting.CD34+ cells were immunologically selected in 11 SSc patients, 2 dermatomyositis patients and a WG patient just after apheresis.All of the patients were treated with high-dose CY $(200 \mathrm{mg} / \mathrm{kg})$ and received auto-HSCT.There was no treatment-related mortality. As toxicity, there were a variety of posttransplant infections such as adenoviral hemorrhagic cystitis, herpes zoster and cytomegaloviral antigenemia.In patients with SSc, skin sclerosis was

and the improvement was sustained for60 months after auto-HSCT. Vital capacity was significantly increasedat 48 months after HSCT and KL-6, a marker for IP, was significantly decreased during 12-60 months after HSCT. A titer of anti-Scl-70 was significantly decreased during 1-60 months after HSCT. Progression-free and overall 5-year survivals were $66 \%$ and $93 \%$, respectively. In patients with dermatomyositis, progressive IP and skin ulcers were dramatically improved after auto-HSCT. In a patient with WG, the size of the left orbital granuloma decreased.To study the mechanism for durable effects, we analyzed the immune reconstitution after HSCT in 11 SSc patients with CD34+ selection. The number of CD8+ $T$ cells recovered as fast as a month, in contrast,that of CD4+ $T$ cells was severely suppressedfor 60 months after HSCT. The number of CD4+CD45RO+ memory $T$ cells recovered earlier than those of CD4+CD45RA+ naïve $T$ cells. The number of $B$ cells recovered to the baseline at 12 months after HSCT. Of note, CD4+ Th1 cells became predominant, and Th1/Th2 ratio was significantly increased during 1-60 months after HSCT. CD34-selected auto-HSCT was more effective on skin sclerosis and was more strongly associated with viral infection than unmanipulated autoHSCT. In conclusion, auto-HSCT is feasible and effective in the treatment of severe AD.
\end{abstract} markedly improved in all of the patients within 6 months 


\title{
The use of Mesenchymal Stromal Cells to modulate Immune Responses: Risk and Benefit
}

\author{
Willem E Fibbe*
}

\begin{abstract}
Mesenchymal stem cells (MSCs) are multipotent progenitor cells that have emerged as a promising therapeutic modality for tissue regeneration and repair. The interest in MSC therapy has been further raised by the observation that MSCs are able to modulate immune responses in vitro and in vivo. These properties may be used in clinical therapy in the context of allogeneic stem cell transplantation or treatment of auto-immune disorders. MSCs are known to secrete a number of cytokines and regulatory molecules implicated in regulation of hematopoiesis. These characteristics have generated clinical interest to use MSCs to enhance hematopoietic stem cell engraftment. MSCs suppress the proliferation of $\mathrm{T}$ cells induced by alloantigens or mitogens and MSCs have been reported to induce $T$ cell division arrest, to inhibit the differentiation and maturation of dendritic cells, and to decrease the production of inflammatory cytokines. Allogeneic MSCs may prolong skin allograft survival in immunocompetent baboonsand may prevent the rejection of allogeneic tumor cells in immunocompetent mice. The mechanisms underlying these effects of MSCs have not been clearly identified.

MSC therapy is associated with several potential risks, including immunogenicity, malignant transformation and ectopic tissue formation. Although initial publication suggested the possibility of human MSCs in culture to

transform into malignant cells, recent evidence indicates that such transformation in-vitro is an extremely rare event. Prolonged expansion of MSCs in-vitro does not result in emergence of independent clones and cytogenetic analysis or comparative genomic hybridisation studies do not reveal the structural abnormalities. Altogether, the experimental and clinical data do not support the possible malignant transformation as a result of prolonged expansion in culture.

In pilot studies, encouraging results have been reported for patients with graft rejection after haplo-identical stem cell transplantation and steroid resistant graft-versus-host disease (GVHD), treated with bone marrow-derived and exvivo expanded MSCs. A majority of the patients (70\%) showed a clinical response on MSC treatment and this response was associated with improved survival (Le Blanc et al. 2008). A recent follow-up study performed in children transplanted in Pavia (Italy) and Leiden (the Netherlands) suggest that the interval between onset of GHVD and treatment with MSCs may be an important variable determining response to MSC treatment. Early MSC treatment after the diagnosis or steroid refractory acute GVHD was associated with a higher probability of obtaining complete remission. These preliminary data may indicate that timing of MSC treatment is a critical determinant of efficacy and outcome.
\end{abstract}




\title{
Proceedings of the 2 nd Meeting of the Asian Cellular Therapy Organization
}

\section{Nonhomologous Hematopoietic Cell Therapy}

\author{
Edwin M. Horwitz *
}

\begin{abstract}
Bone marrow transplantation is accepted as an effective therapeutic modality for genetic and acquired diseases of the hematopoietic system. This cellular therapy is founded on the principle that bone marrow contains hematopoietic stem cells that can repopulate the blood. Over the past two decades, we have recognized that bone marrow also contains mesenchymal stem/progenitor cells. In principle, then, marrow transplantation as a means of transplanting mesenchymal cells could be used to treat disorders of mesenchymal tissues, in an analogous fashion to hematopoietic cell therapy. Using osteogenesis imperfecta (OI), a genetic disorder of bone, as a model system, we demonstrated that transplantation of unmanipulated bone marrow could functionally engraft in recipient bone and provide measurable clinical benefits. Based on the notion that mesenchymal stromal cells (MSCs) generated the donor-derived osteopoiesis, we subsequently intravenously infused allogeneic, gene-marked MSCs obtained frommarrow donors. Measured engraftment of the genemarked MSCs in bone wasexceedingly low; nonetheless, it was associated with an acceleration of growth in the children. Animal models of osteopoietic engraftment and differentiation suggest that nonadherent marrow cells (NAMBCs) are superior to MSCs forosteopoietic engraftment and differentiation to osteoblasts as systemic

osteopoietic engraftment may be an important factor governing the durability of the osteopoietic graft. This animal data led to our third clinical trial which unambiguously demonstrated that marrow mononuclear cell "boosts" in our Ol children can lead to osteopoietic engraftmentand striking clinical benefits in a subset of patients suggesting potent mesenchymal progenitor activity. Moreover, these data suggest that the therapeutic benefit of marrow mononuclear cell therapymay be collagen-mutation dependent. In an animal model of OI, we demonstrate that the NABMCs engraft and differentiate to osteoblasts contributing normal collagen to the OI bone matrix, consistent with our clinical data suggesting a collagen-mutationdependent mechanism. Additional murine data suggests that the cytokine therapy may be able to induce osteopoietic differentiation of donor marrow cells after BMT. Our clinical trials and murine models collectively suggest that bone marrow cell therapy is an effective strategy for the treatment of $\mathrm{Ol}$ and possible other disorders of bone. The development of broadly applicable marrow cell therapy for bone will depend, in part, on better knowledge of the transplantation biology of marrow cells and osteopoietic differentiation, which will be most effectively uncovered by both innovative laboratory research as well as scientifically-based clinical trials.
\end{abstract} cell therapy of bone. Moreover, the mechanism of 


\title{
Proceedings of the 2 nd Meeting of the Asian Cellular Therapy Organization
}

JSRM Code: 007030700032

\section{New strategy of adoptive-immunotherapy using $\gamma \delta \mathrm{T}$ cells}

\author{
Hirohito Kobayashi ${ }^{*}$
}

\begin{abstract}
Human gamma-delta T cells $(\gamma \delta$ T cells), which are subgroups of peripheral blood $T$ cells comprising less than $10 \%$ of peripheral blood mononuclear cells (PBM), are known to defend the body against infection. Seventy percent of peripheral blood $\gamma \delta$ T cells express $\mathrm{V} \gamma 2$ and $\mathrm{V} \delta 2$ from among the variable elements of $T$ cell receptors $(\mathrm{V} \gamma 2 \mathrm{~V} \delta 2 \mathrm{~T}$ cells) and these $\mathrm{V} \gamma 2 \mathrm{~V} \delta 2 \mathrm{~T}$ cells are activated in a TCR-dependent manner by several small phosphorylatedor aminated alkyl molecules. We have synthesized more than 60 kinds of monoethylpyrophosphate derivatives and 2-methyl-3-butenyl-1pyrophosphate (2M3B1PP) which exhibit potent stimulation of $\gamma \delta \mathrm{T}$. cells more than 100-fold that of isopentenyl pyrophosphate (IPP) which is one of the natural ligands of $\mathrm{V} \gamma 2 \mathrm{~V} \delta 2 \mathrm{~T}$ cells. We also generated in vitro culture systems of $\mathrm{V} \gamma 2 \mathrm{~V} \delta 2 \mathrm{~T}$ cells, and these cultured $\mathrm{V} \gamma 2 \mathrm{~V} \delta 2 \mathrm{~T}$ cells showed a strong anti-tumor effect.

Many tumor cells up-regulate the mevalonatepathway. IPP which is one of the intermediate metabolisms of the pathway accumulates in tumor cells and $\mathrm{V} 2 \mathrm{~V}$ 2. cells and recognizes IPP of the TCR and exhibitscytotoxity.Nitrogen containing bisphosphonates, such as zoledronic acid (Zol), inhibit Farnesylpyrophosphate (FPP) synthetase which is one of the

important enzymes in the mevalonate pathway. Inhibition of FPP synthetase resulted in accumulation of IPP in the tumor cells which $\mathrm{V}_{\gamma} 2 \mathrm{~V} \delta 2 \mathrm{~T}$. cells recognize and then kill them.

We conducted a phase $1 /$ llaclinical trial of adoptive transfer of $\mathrm{V} 2 \mathrm{~V} 2 \mathrm{~T}$ cells to patients with advanced renal cell carcinoma. Eleven patients who had undergone nephrectomy and had lung metastasis were enrolled. Peripheral blood $\mathrm{V} \gamma 2 \mathrm{~V} \delta 2 \mathrm{~T}$ cells obtained from the patients were stimulated ex vivo with 2M3B1PP and transferred in combination with Zol and recombinant human Interleukin-2 (IL-2). The proportion of peripheral blood $\mathrm{V} \gamma 2 \mathrm{~V} \delta 2 \mathrm{~T}$ cells among $\mathrm{CD}^{+}$cells typically peaked three to five days after transfer. Tumor doubling time was prolonged in all patients, and the best overall responses were $1 \mathrm{CR}, 5 \mathrm{SD}$, and $5 \mathrm{PD}$ as defined based on the RECIST criteria. While ten patients developed adverse reactions of grade 3 and most symptoms swiftly reverted to normal during the course of the treatment. In conclusion, this clinical trial demonstrated that our regimen for the adoptive transfer of $\mathrm{V} \gamma 2 \mathrm{~V} \delta 2 \mathrm{~T}$ cells in combination with Zol and IL-2 was well tolerated, and that objective clinical responses could be achieved in some patients with advanced renal cell carcinoma. These results demonstrated the safety and feasibility of $\mathrm{V} \gamma 2 \mathrm{~V} \delta 2 \mathrm{~T}$ cell immunotherapy for renal cell carcinoma.
\end{abstract}




\title{
Proceedings of the 2 nd Meeting of the Asian Cellular Therapy Organization
}

JSRM Code: 007030700033

ISSN $0973-7154$

\section{Autologous T cell Therapy Against Tumors}

\author{
Jaeseung $\operatorname{Lim}^{*}$
}

\begin{abstract}
Adoptive immunotherapyadministrating autologous or allogeneic ex vivoexpanded cells after isolation of antigen specific cells, is a promising approach to inducingantitumor immune responses. Recent studies have shown the effectiveness of immune celltransfertherapies for the treatment of patients with selected metastatic cancers.

Among lots of effector cells for cancer treatment, cytokineinduced killer (CIK)cells are shown to be a heterogeneous population, and themajor population expresses both the $\mathrm{T}$ cell marker CD3 andthe NK cell marker CD56. Also, CIK cells have potent cytolytic activity and exhibits non major histocompatibility complex $(\mathrm{MHC})$ restricted lysis of target tumor cells.

Our products, Immuncell-LC $C^{\mathrm{R}}$ which is derived from peripheral blood mononuclear cells and cultured under well defined culture conditions for 2-3 week, has characteristics

bearing cytotoxic $T$ cells $(C T L)$ and CIK cells. We have recently reported that Immuncell-LC ${ }^{R}$ could suppress the growth ofa variety of solid tumors including HCC tumor in animals or ex vivo effectively. Moreover, many reports showed that CIK treatment for $\mathrm{HCC}$ was effective and safe. Now, we are under Phase III clinical trial to evaluate efficacy and safety of Immuncell-LC ${ }^{R}$ for HCCpatients undergone curative hepatic resection. In addition, Immuncell-LC ${ }^{R}$ is challenged to brain tumors such as glioblastoma, median survival time of which is poor due to the inevitable recurrence.

Today, I will present introduction of Immuncell-LC ${ }^{R}$ for adoptive immunotherapy including our preclinical results and clinical design and our developments to improve immunotherapy efficacy for cancer.
\end{abstract}




\title{
Ex vivo-expanded allogeneic natural killer cells for clinical application in anti-tumor immunotherapy
}

\author{
Yu-Kyeong Hwang *
}

Abstract

\begin{abstract}
A novel method of expansion and activation of natural killer (NK) cells for clinical application under good manufacturing practice (GMP) compliance was established. Purified NK cells from $100 \mathrm{~mL}$ of peripheral blood were stimulated with interleukin-2 (IL-2) and anti-CD3 antibody (OKT-3) in a closed culture bag system for 14 days. More than $1 \times 10^{9}$ of highly pure population of $\mathrm{CD} 16^{+} / \mathrm{CD} 56^{+} \mathrm{NK}$ cells were obtained while contaminants such as $\mathrm{T}$ cells, B cells, or monocytes were analyzed by less than 0.5 percent.

Expanded NK cells showed potent efficacy on killing tumor cells. In response to target cells, effector cytokines such as IFN- and TNF- were efficiently secreted 10-fold more compared to resting NK cells. Adhesion molecules, DNAM-1; activating receptors, NKG2D; and natural cytotoxicity receptors (NCRs), NKp30, NKp44, and NKp46 were up-regulated by ex vivo-expansion resulting in the enhancement of cytolytic activity against tumor cells. This potent cytotoxicity of expanded NK cells revealed a more apparent result with perforin/granzyme-dependent necrosis over than TRAIL or FasL-mediated apoptosis of target cells. In addition, expanded NK cells effectively
\end{abstract}

discriminated tumor cells from allogeneic normal peripheral blood lymphocytesand selectively killed those transformed cells.

For non-clinical testing for toxicity and kinetics, human NK cells were adoptively transferred into SCID mice via tail vein. The administrated NK cells were mainly found in the lung, liver, and spleen for 48 hours and a few were circulated in the blood stream. One week after transfer, human NK cells were hardly detected by immunohistochemistry, flow cytometry or PCR. There were no reports concerning any significant adverse effects of NK cell transfusion.

In conclusion, an innovative process of large-scale expansion of NK cells with up-regulated expression of cytotoxic effector molecules was developed. These ex vivoexpanded allogeneic NK cells were demonstrated to be safe and are expected to be effective for clinical application in anti-tumor immunotherapy.

"This study was supported by a grant of the Korea Healthcare technology R\&D Project, Ministry of Health and Welfare, Republic of Korea. (A062260)." 


\title{
Proceedings of the 2 nd Meeting of the Asian Cellular Therapy Organization
}

\section{Dendritic Cell Immunotherapy of Gastrointestinal Tumors}

\author{
H. Kim Lyerly ${ }^{*}$
}

\begin{abstract}
Metastatic colorectal cancer remains lethal in the majority of patients despite effective chemotherapy and monoclonal antibodies, underscoring the need for additional therapies. Infiltration of tumor by $\mathrm{T}$ cells has been associated with longer survival of colorectal cancer patients in numerous studies. This finding leads to the hypothesis that adaptive immunity is capable ofcontrolling colorectal cancers and that activation of $\mathrm{T}$ cell responses against colorectal cancers (using active specific immunotherapy or "therapeutic cancer vaccines") results in clinical benefit. Indeed, various vaccination strategies have been reported to increase $T$ cell infiltration into murine and human tumors including colorectal cancer. We have performed preclinical studies and $\mathrm{NCl}$-sponsored clinical trials demonstrating that autologous DC modified with viral vectors encoding tumor antigens induce more potent $T$ cell responses than DC loaded with peptides or conventional mRNA. We developed a broadly applicable and clinically feasible strategy to modify autologous DC using a new generation of recombinant pox vectors encoding a tumor antigen and TRICOM (triad of co-stimulatory molecules B7.1, ICAM-1, LFA-3). Early clinical studies demonstrated safety and immunogenicity. We have completed an NCl-funded, multicenter, randomized phase II clinical trial of heterologous prime-boost vaccination with recombinant pox vectors

encoding CEA and MUC-1 plus injection site doses of GMCSF versus heterologous prime-boost vaccination with autologous DC loaded ex vivo with pox vectors for patients with resected hepatic and pulmonary metastases of CRC $(n=74)$. The purpose of the study was to "pick a winner" between either the DC loaded with the poxvectors or the poxvectors given with GM-CSF to choose the strategy to be used in a phase III trial. A preplanned endpoint was to determine overall survival for the vaccine strategies and also, to compare this survival with that of a contemporary control group of similar patients who were not vaccinated. We demonstrated improved overall survival in patients with metastatic CRC vaccinated against carcinoembronic antigen (CEA) and MUC1 compared to non-vaccinated controls. Notably, this strategy was effective in the setting of minimal tumor burden following complete metastasectomy. Unfortunately, the majority of patients are unsuitable for complete metastasectomy and will not only have detectable tumor, but also suffer from significant immunologic dysfunction, partly characterized by significant numbers of regulatory $T$ cells ( $\left.T_{\text {regs }}\right)$. Consequently, we hypothesize that patients will benefit from cancer immunotherapy in the setting of minimal tumor burden, or in combination with immunomodulatory strategies that circumvent the immunologic dysfunction.
\end{abstract}




\title{
Proceedings of the 2nd Meeting of the Asian Cellular Therapy Organization
}

\section{Immunotherapy for the Treatment of Advanced Prostate Cancer}

\begin{abstract}
Mark W. Frohlich *
Abstract

Sipuleucel-T is an autologous active cellular immunotherapy designed to stimulate an immune response to prostate cancer. It is FDA approved for the treatment of men with asymptomatic or minimally symptomatic castrate resistant prostate cancer, based on prolongation of overall survival. Sipuleucel-T is manufactured by culturing a patient's peripheral blood mononuclear cells, including antigen presenting cells, with a recombinant protein comprising a tumor-associated antigen (prostatic acid phosphatase) and granulocyte-macrophage colony stimulating factor. Treatment consists of 3 infusions at approximately 2 -week intervals, resulting in a prime-boost pattern of immune activation, with a robust antigen-specific cellular and humoral immune response. Specifically, antigen presenting cells are activated after culture with the recombinant protein, as evidenced by an increase in CD54

expression in the first dose at Week 0 , that is further upregulated in subsequent doses at Week 2 and Week 4. Activated APC-associated cytokines and chemokines are produced in the media during manufacture of sipuleucel-T, and activated T-cell-associated cytokines are detected in the media of the second and third products. Antigenspecific T-cell proliferative and IFN gamma ELISPOT memory responses are evident after administration of the first sipuleucel-T dose. There are correlations between product parameters as well as peripheral immune responses and overall survival. .Adverse events are generally mild to moderate and resolve within 2 days. As the first autologous cellular immunotherapy to demonstrate a survival benefit, sipuleucel-T is an important new treatment for men with asymptomatic or minimally symptomatic castrate resistant prostate cancer.
\end{abstract}




\title{
Clinical translation of Chimeric Antigen Receptor (CAR) messenger RNA (mRNA) loaded T- and NK-cell immunotherapy for safe, tumor-targeted bioactivity
}

\author{
Madhusudan V. Peshwa
}

Abstract

\begin{abstract}
In recent publications, human proof of concept for Chimeric Antigen Receptor (CAR) engineered $T$ cells was demonstrated in a pilot study with CLL patients.[1,2] Three patients were treated with expanded autologous $T$ cells virally-transfected with a CAR molecule that is specific for recognition of the CD19, a molecule expressed solely on B-cells and on leukemia / lymphoma cells. Twelve months subsequent to treatment, two of the patients exhibit Complete Response and one patient exhibits Partial Response with $>90 \%$ reduction in tumor burden.

Recognizing the safety and toxicity of virusmediated delivery of CAR, MaxCyte has developed an alternative approach to introducing the CAR as an messenger RNA (mRNA) molecule and using MaxCyte cell loading technology platform to enable a robust, cGMP compliant, method of manufacturing for clinical / commercial delivery of CAR-engineered T- and NK-cells.

In collaborations with investigators at the University of Pennsylvania (Philadelphia, PA), using CD19 targeted CAR mRNA loading into ex vivo expanded $T$ cells, it has been demonstrated that the in vitro and in vivo antitumor activity of a-CD19 CAR mRNA loaded T cells is equivalent to that of $T$ cells transfected with $\alpha-C D 19$ CAR encoded by lentiviral vectors.[3] Similar pre-clinical studies using a-Mesothelin CAR mRNA loaded T cells demonstrate safety and anti-tumor efficacy directed against tumors that specifically over-express Mesothelin;[4] a tumorassociatedantigen preferentially expressed on multiple solid tumors including mesothelioma, ovarian cancer, pancreatic cancer and GI cancers. The safety and therapeutic activity of $\alpha-$.
\end{abstract}

Mesothelin CAR mRNA loaded $T$ cells is under investigation in human clinical studies at the University of Pennsylvania

In parallel collaborations with investigators at the St Jude Childrens Research Hospital (Memphis, TN), using CD19 targeted CAR mRNA loading into ex vivo expanded NK cells, it has been demonstrated that the in vitro and in vivo anti-tumor activity of a-CD19 CAR mRNA loaded NK cells is equivalent to that of $T$ cells transfected with $\alpha-C D 19$ CAR encoded by retroviral vectors.[5] These pre-clinical studies form the basis for filing of an IND application with the US FDA, for initiation of human clinical trials to evaluate the safety and therapeutic activity of $\alpha$-CD19 CAR mRNA loaded NK cells for treatment of AML in pediatric population.

The presentation will provide a summary of translational experiences leading to the IND filings for Chimeric Antigen Receptor (CAR) messenger RNA (mRNA) loaded T- and NK-cell immunotherapy

\section{References:}

1. Porter, DL, et. al., N. Engl. J. Med. (2011). DOI: 10.1056/NEJMoa1103849

2. Kalos M, et. al., Sci. Transl. Med. (2011). DOI: 10.1126/scitransImed.3002842

3. Barrett DM, Zhao Y, et. al., Hum. Gene Ther. (2011). DOI: 10.1089/hum.2011.070

4. Zhao Y, et. al., Cancer Res. (2010). DOI: 10.1158/0008-5472.CAN10-2880

5. Li, L, et. al., Cancer Gene Ther (2010). DOI: 10.1038/cgt.2009.61 


\title{
Proceedings of the 2 nd Meeting of the Asian Cellular Therapy Organization
}

\section{Systemic MSC Therapy for Genetic and Acquired Orthopedic Disorders}

\author{
Satoru Otsuru
}

Abstract

\begin{abstract}
Mesenchymal stromal cells (MSCs), frequently termed mesenchymal stem cells, are one of the most commonly investigated cells in cell therapy. We reported the first clinical trial of allogeneic MSC infusions in a study of MSC therapy for children with osteogenesis imperfecta (OI), a genetic disorder of collagen type I. The children showed an acute acceleration of growth following the cell therapy. Curiously, despite the striking clinical outcome, very low levels of donor MSCs were detected in the patients' bone suggesting that the mechanism underlying the clinical effect of MSC therapy is unrelated to osteopoietic differentiation. We reasoned that since bone growth is initiated by chondrocytes in the growth plate (GP), MSCs may be directly acting at GP. However, in our murine model, MSCs do not engraft and differentiate to chondrocytes in the GP. Moreover, freshly isolated primary chondrocytes cocultured with MSCs do not proliferate greater than controls suggesting that MSCs do not secrete a soluble mediator that directly stimulates GP chondrocyte activity. We then considered that the growth may be the result of a complex metabolic pathway initiated by a MSCsecreted soluble mediator. To test this hypothesis, we infused mice with MSCs and obtained serum at day 7. Chondrocytes assayed in media supplemented with serum
\end{abstract}

taken from MSC-infused mice stimulated greater chondrocytes proliferation than controls $(P<0.01)$. Analysis of proliferating cell nuclear antigen (PCNA) in the GP of mice infused with MSCs revealed significantly more proliferating cells compared to controls $(P=0.006)$. To determine whether our findings were applicable to OI, we transplanted MSCs into OI mice. Four weeks after the transplantation, we observed a significantly increased lumbar vertebral length $(P=0.0008)$ as well as total body weight $(P=0.0025)$. Based on these findings, we hypothesized that MSC therapy may protect articular cartilage (AC) from developing osteoarthritis (OA) by stimulating the proliferation of chondrocytes in the AC. To examine this idea, we transplanted MSCs into a murine model of OA once per week for four weeks, beginning at the time of $\mathrm{OA}$ induction. The joints were then evaluated with the Osteoarthritis Research Society International (OARSI) scoring system. The joints treated with MSCs resulted in significantly a lower score than controls $(\mathrm{P}<0.0001)$ with proliferating chondrocytes evident in the AC. Our data indicate that the mechanism of both MSCstimulated linear growth and delayed development of $O A$ is the secretion of a soluble factor that initiates a metabolic pathway resulting in chondrocyte proliferation in GP and AC. 


\title{
Mechanisms of MSC (mesenchymal stem cell) accumulation at the sites of tumors
}

\author{
Ryosuke Uchibori, Keiya Ozawa*
}

\begin{abstract}
Background: MSCs have the ability to accumulate at the sites of tumors when injected into tumor-bearing mice, and therefore MSCs are considered to be an appropriate cellular vehicle for cancer-targeted gene therapy.

Aims: Although various growth factors and chemokines may be involved, the detailed molecular mechanisms of MSC accumulation at tumors are poorly understood. In this study, we focused on MSC-endothelial cell (EC) adhesion following TNF- $\alpha$ stimulation as a clue to elucidate the mechanisms.

Methods: To assess tumor tropism of MSCs, GFPexpressing MSCs or fibroblasts were injected into the left ventricular cavity in tumor-bearing nude mice. Luciferaseexpressing MSCs or fibroblasts were also injected to the mice in order to periodically trace the cells using an in vivo imaging system (IVIS). Migration capacity of MSCs and fibroblasts was examined by transwell migration assay. The MSC adhesion to ECs was tested with or without TNF- $\alpha$.

stimulation in vitro. Luciferase-expressing MSCs were injected to the mice treated with parthenolide, and IVIS imaging was periodically performed

Results: Injected MSCs were detected at tumor lesions, but fibroblasts were not. On the other hand, the factors that induced MSC migration in vitro enhanced fibroblast migration as well. Interestingly the stimulation of MSCs with TNF- $\alpha$ enhanced MSC adhesion to ECs in vitro. This adhesion was partially inhibited by blocking antibodies against VCAM-1 and VLA-4. The similar phenomenon was not observed for fibroblasts. Furthermore, in the mice treated with parthenolide, MSC accumulation at the tumors was significantly inhibited.

Conclusions: Our data suggest that the MSC-EC adhesion following activation by TNF- $\alpha$ may play a crucial role in MSC accumulation at tumor lesions. The present findings would be important to develop efficient MSC-based cancer gene therapy.
\end{abstract}




\title{
Oncolytic measles virus-loaded human bone marrow- derived mesenchymal stem cells as systemic cancer therapeutic agents for human hepatocellular carcinoma
}

\author{
Ong HT, Federspiel MJ, Russell SJ,Peng KW and Hui KM*
}

\begin{abstract}
Hepatocellular carcinoma (HCC) represents one of the major global health concerns affecting both developing and developed nations. Though currently hepatectomy and orthotopic liver transplantation could provide a potentially curative treatment for HCC, only a small proportion of patients will be eligible for these treatments since HCC is often detected at an advanced stage. Additionally, the observed cure rates for these treatments are elusive due to the high incidence of recurrence and salvage therapies for recurrent disease are not curative. Hence, the development of innovative therapeutic platforms for clinical applications is urgently needed. An emerging cancer therapeutic tool is the mesenchymal stem cell (MSC)-based therapy. MSC is a potentially good candidate due to its ability to target tumor cells and integrating into the stroma. Their easiness of expansion in culture is an additional advantage. Recently oncolytic measles viruses (MV) have been demonstrated to have potent oncolytic activity against several types of human cancers and their toxicity has also been reported to be minimal. Oncolytic MV targets CD46+ cells. MV uses the hemagglutinin envelope glycoprotein to infect cancer

glycoprotein to trigger fusion of the viral cell membranes for virus entry. Expression of these fusogenic hemagglutinin and fusion proteins on surfaces of virus-infected cells results in massive intercellular fusion with uninfected neighbouring CD46+ to generate the characteristic MVinduced cytopathic effects of syncytia formation. We have demonstrated that most of the human HCC tested are CD46+. We are therefore exploiting the ability of the Edmonston vaccine lineage of MV as a CD46+-selective oncolytic agent for HCC cancer therapy. In this presentation, the homing ability of MSC was investigated in an orthotopic human HCC xenograft model. Results showed that systemic delivery of oncolytic MV-loaded human bone marrow (BM)- derived MSC preferentially home at tumor sites and the overexpression of CD46 on cell surfaces of human HCC cells resulted in the preferential killing of the tumor cells. Pre-existing antiviral antibodies in cancer patients can potentially neutralize the MV and decrease its antitumor potency. Hence, the ability of the BM-derived MSC to protect the "naked" MV viruses from antibody neutralization is also being investigated.
\end{abstract} cells via the cellular CD46 receptor and the fusion envelope 


\section{Phase-I trial of T cell-depleted haplo-identical stem cell transplantation (Haplo-SCT) combined with HSV-TK gene modified $T$ cell add-back}

Yuji Heike*, Takako Wakeda, Takahiro Fukuda, Shinichiro Mori, IkueiNukaya, Jun-ichiMineno, Kazuto Takesako and Yoich Takaue

\begin{abstract}
In Japan, cord blood is the first choice as an alternative donor of hematopoietic stem cell transplantation (HSCT) for high-risk leukemia patients, when appropriate donors (HLAfull match or 1 locus mismatch donors) are not available. However, there are several problems in cord blood transplantation (CBT), especially in early phase of transplantation, such as higher infection and relapse rate and late recovery of platelet, etc. Haplo-SCT is another alternative method and has been developed in many countries. The problem of naked haplo-SCT is severe graft- versus-host disease (GvHD) caused by uncontrolled allo-reactivity of donor T cells and that of T-cell depleted haplo- SCT is higher infection and higher relapse rate caused by delay of immune reconstitution (IR).
\end{abstract}

Abstract

We are challenging T-cell depleted Haplo-SCT combined with HSV-TK gene modified T cell add-back therapy and we had experiences of the best-case scenario and the worst-case scenario in the first two cases.

The first case is a $47 \mathrm{y} .0$. female ALL patient and IR was achieved after three times T-cell "add-back". GvHD appeared on the same day of IR and administration of GCV completely cured GvHD. This patient is alive without relapse or viral/fungal infection for 12 months. The second case is a 59 y.o. male AML patient and he died from viral infection before achievement of IR. These two cases suggested that add-back therapy with HSV-TK gene modified T cells is essential for successful Haplo-SCT, and HSV-TK/GCV system is useful to control GvHD caused by addbacked T cells.

I will show the detail of the cases and discuss the benefits of T-cell depleted Haplo-SCT combined with HSV-TK gene modified T cell add-back therapy. 


\title{
Proceedings of the 2 nd Meeting of the Asian Cellular Therapy Organization
}

JSRM Code: 007030700042

\section{Engineered T Cell Immunotherapy for Cancer in Japan}

\author{
Kazuto Takesako*
}

\begin{abstract}
Recently there are several big findings in the field of engineered $\mathrm{T}$ cell immunotherapy for cancers and viral infections using $\mathrm{T}$ cell receptor (TCR) and others. The Gene Medicine Unit of Takara Bio Inc. is focusing on the development of such engineered $\mathrm{T}$ cell immunotherapy for cancer and HIV infection. Our proprietary technologies are being used for these developments, including gene transduction method using RetroNectin, a recombinant human fibronectin fragment, siRNA technology for high expression of TCR genes in T cells, and our genes such as MazF, E. coli RNase. In the TCR gene therapy, we are now focusing on the use of MAGE-A4 or WT1 tumor antigen-

and planning several clinical studies to treat cancer patients, in which TCR gene-modified autologous T cells are used for esophageal cancer or hematological malignancies, and HSV-TK (Herpes Simplex Virus thymidine kinase) gene-modified donor $\mathrm{T}$ cells are used for hematological malignancies. Also, we will perform Takarasponsored clinical trials to use the gene-modified T cells as pharmaceuticals after testing for the quality and storing at a stable condition. In the future, we will go forward to perform such clinical trials to determine a better method to give better efficacy in the treatment of cancer patients in Asia including China and Korea.
\end{abstract} specific, HLA-A24 restricted TCR. In Japan, we are doing 


\title{
Proceedings of the 2 nd Meeting of the Asian Cellular Therapy Organization
}

\section{Adoptive T-Cell Therapy for Cancer from the Benchtop to the Bedside and bevond}

\author{
Renier J Brentjens*
}

\begin{abstract}
$\mathrm{T}$ cells may be genetically modified to target tumor associated antigens through the retroviral introduction of genes encoding artificial T cells receptors, termed chimeric antigen receptors (CARs), specific to these targeted antigens. We have previously demonstrated in xenotransplant models of systemic human CD19+ B cell tumors in immunecompromised SCID-Beige mice, that human $T$ cells engineered to express the 19-28z CAR specific to the B cell antigen CD19 can efficiently eradicate disease. Based on these findings we have initiated clinical trials treating patients with relapsed or refractory chronic lymphocytic leukemia (CLL) of B cell acute lymphoblastic leukemia (B-ALL). Meanwhile, we furthered out pre-clinical studies by generating a transgenic C57BL6(mCD19-/hCD19+/- ) immune competent modified syngeneic $\mathrm{T}$ cell murine mouse model. Studies using this tumor model

conditioning chemotherapy to allow for eradication of systemic EL4(hCD19) tumors and predicted normal B cell aplasias. Based on these findings we modified our clinical trials to include prior cyclophosphamide conditioning. Promising clinical trial results from our institution will be presented and compared to recently published clinical trial results from other institutions (the National Cancer Institute, and the University of Pennsylvania) similarly utilizing autologous $T$ cells modified with CD19-targeted CARs. Finally, preclinical studies will be presented demonstrating enhanced efficacy of tumor targeted $\mathrm{T}$ cells additionally modified to express the IL-12 cytokine gene. These studies demonstrate potential enhanced anti-tumor efficacy in the absence of prior conditioning chemotherapy and may hold promise for a more robust anti-tumor effect in future clinical trials.
\end{abstract} demonstrate requisite need for prior cyclophosphamide 


\title{
Proceedings of the 2 nd Meeting of the Asian Cellular Therapy Organization
}

JSRM Code: 007030700044

ISSN $0973-7154$

\section{Cartilage Repair -Past, Present and Future}

\author{
Mitsuo Ochi*
}

\begin{abstract}
Several surgical approaches to repair cartilage defects have been reported such as reattachment of a detached osteochondral fragment to the lesion, microfracture, mosaicplasty and $\mathrm{ACl}$. We treated eight cartilage defects with meniscal transplantation from 1990 to 1995. Then we started to perform transplantation of tissue-engineered cartilage made ex vivo for the treatment of osteochondral defects of the joints (110 cases) as a second generation of chondrocyte transplantation from 1996. Sixty knees who had received transplantation of tissue-engineered cartilage for cartilage defects were followed up for at least 5 years. Although the clinical results were satisfactory, we need the

less invasive technique.

One of the less invasive surgical procedures to treat large cartilage defects is microfarcture or drilling. However, these techniques under arthroscopy are not sufficient to repair cartilage defects with hyaline cartilage. I think that there are two weak points such as insufficient number of mesenchymal stem cells and early overloading on the treated area. I would like to show our novel approaches using an external magnetic field to deliver precisely injected cells with magnetic beads to an articular defect and articulated distraction device for reducing the load.
\end{abstract} surgical approaches to treat large cartilage defects with 


\title{
Strategies for addressing stand-alone corneal epithelial and endothelial diseases using in vitro expanded cells using Nano scaffolds and polymers
}

\begin{abstract}
Abraham $S^{*}$
Abstract

Corneal diseases are one of the major causes of total or partial blindness in developing countries. The number of patients registered for cadaver corneal transplantation in India alone is close to 100000 per year. According to the registries, $90 \%$ of the patients are unable to get a transplantation done, due to lack of donor corneas. When the data on the disease pattern of such patients was analyzed, nearly one-third had stand-alone endothelial disease, one-third epithelial disease and the remaining one-third only had a total damage of the cornea requiring a pan keratoplasty per se. Therefore we have come with solutions for addressing the corneal endothelial and epithelial diseases using novel nanomaterial based in vitro expanded corneal limbal stem cells and corneal endothelial precursors respectively.

Solution for unilateral corneal epithelial diseases: Persistent corneal ulcer has been a common disease in the developing countries and several scaffolds have been used to grow the opposite eyes corneal limbal tissue stem cells in vitro for transplantation to the affected portion of the eye. We have tried a novel thermoreversible gelation polymer for the in vitro expansion and the same when tried in animal models, has proven to be successful by all given clinical parameters and also histopathologically, confirming

the regeneration of the experimentally damaged epithelium including the limbus. Avoiding biological materials such as human amniotic membrane has been thus possible with this method and in our methodology we have proven that the in vitro grown cells when transplanted along with apolymer scaffold which allows a free movement of the cells within over the damaged cornea works, instead of a sheet-type corneal cells grown in vitro.

Solution for unilateral corneal endothelial diseases: Endothelial keratoplasty to treat stand-alone corneal endothelial disease in patients with normal stroma and corneal epithelium is an established clinical procedure in which one-donor-endothelium helps treat the vision of onepatients eye. We have successfully transported endothelial buttons separated from donor corneas embedded in a polymer cocktail without cool preservation to a central lab taking maximum of $72 \mathrm{Hrs}$. Then we expanded the endothelial precursors in the lab as previously reported in the literature followed by our own methods and then transplanted the cells on to the corneal endothelium of bulls eye using a nanocomposite gel sheet with successful engraftment, thus paving way for a clinical application treating many eyes with one donor corneal endothelium, what we call as "An eye for Eyes" mission.
\end{abstract}




\title{
Proceedings of the 2 nd Meeting of the Asian Cellular Therapy Organization
}

\section{Recent advances in clinical application of human stem cells}

\author{
Kwang-Hoe Chung ${ }^{*}$, Hyung-Min Chung, Dong Ryul Lee, Jisook Moon, Kwang-Yul Cha
}

\begin{abstract}
Stem cells have two important properties that distinguish themselves from other types of cells; they can both proliferate without changing their phenotypes indefinitely, and they also can differentiate into one or more new kind of cells depending on the culture conditions. Thus, stem cell therapy could be most effective to treat the diseases that are marked by the loss of cells. In last decade, various types of stem cells have been identified from the preimplantation stage embryos, fetus, placenta, and adult tissues. Moreover, it is now almost a common practice to make induced pluripotent stem cells (iPS) from various adult somatic cells using only a few defined factors.

Recent advances in the stem cell biology, including the development of optimized cell type specific culture systems, and the broader understandings of biochemical and molecular signals involved in cell self-renewal and

differentiation have made the cell based therapy closer to the practical uses. As of now, at least 180 adult stem cell therapies are being used or tested in real life in the forms of bone marrow stem cell, mesenchymal stem cell, cord blood stem cell, fetal brain stem cell and fat stem cell transplantations.

CHA Health Systems, Korea is developing feasible and applicable stem cell therapies for various clinical studies with leading research institutes in ACT Inc, USA. We are now extensively studying human embryonic stem cells (hES), neural stem cells, and various adult stem cells for medical applications. In addition, CHA Health Systems recently developed $\mathrm{CHA}$-StationTM, an integrated cell separation system and established comprehensive human stem cell banks such as germ cell bank, fetus stem cell bank, embryo bank, and adult stem cell bank.
\end{abstract}




\title{
Commercialization of allogeneic, off-the-shelf, adult stem cell drug products using human Umbilical Cord Blood- derived Mesenchymal Stem Cells (hUCBMSCs)
}

\begin{abstract}
Antonio S J Lee *
Abstract

Human cord blood-derived Mesenchymal Stem Cells (hUCB-MSCs) are a robust source for not only giving rise to a new cell type in need but also for secreting various trophic factors which will signal the activation of host's own stem cells for producing required cell types and drive the regenerative process.

MEDIPOST has made R\&D efforts for over 10 years for developing adult stem cell drugs to meet unmet medical needs in areas such as osteoarthritis, stroke, Alzheimer's disease, bronchopulmonary dysplasia (BPD) and improving the efficacy of hematopoietic stem cell transplantation (HSCT).

Through rigorous pre-clinical studies and clinical trials, we

adult stem cell drug materials. The drug products under development include CARTISTEM® for cartilage regeneration which has completed Phase III clinical trial in Korea and is awaiting Biologics License Application (BLA) approval from the KFDA. Once approved, CARTISTEM® will become available as the world's first hUCBMSC derived adult stem cell drug for treating osteoarthritis and will be marketed in Korea in 2012. CARTISTEM® has also received an Investigational New Drug (IND) clearance from the US-FDA in February 2011 and the Phase I/Ila clinical trial will soon commence in the US. Other hUCB-MSC products named PNEUMOSTEM® for treating Bronchopulmonary Dysplasia (BPD) and NEUROSTEM® for treating Alzheimer's Disease (AD) are both undergoing Phase I clinical trials in Korea.
\end{abstract} have proven the safety and efficacy of hUCB-MSCs as 


\title{
Proceedings of the 2 nd Meeting of the Asian Cellular Therapy Organization
}

\section{Generation of Functional Platelets from Pluripotent Stem Cells}

\author{
Hiromitsu Nakauchi*
}

\begin{abstract}
Since the discovery of ABO blood type by Karl Landsteiner, blood transfusion has been used extensively in clinical practice as one of standard therapeutic interventions. Over the century, blood transplantation has been dependent on blood donation, and the risks associated with it such as immune reactions or infections have not been completely resolved. In addition to these problems, changes in social structure leading to low birthrate and aging population in a society like ours have made it difficult to maintain sufficient amount of donated blood constantly. We wondered whether we would still be doing blood transfusion using donated blood 50 years from now and as an alternative to it, we started to search for a possibility of using pluripotent stem cells as a source of blood cells. Our initial target is

Recently, we established an in vitro culture system whereby hESCs or hiPSCs can be differentiated into hematopoietic progenitors within "sac"-like structures, which we termed ES-sacs or iPS-sacs. The cells inside these sacs were capable of differentiating into mature blood cells including megakaryocytes and functional platelets (Takayama et al., Blood, 2008). Furthermore, we found that these human iPSC-derived platelets can form thrombus together with mouse platelets in vivo (Takayama et al. J. Exp. Med. 2010). These results collectively indicated that hESCs and hiPSCs can be potential sources for platelet production and that this system will contribute to understanding the mechanism of various genetic platelet disorders and to development of novel cell/gene therapy.
\end{abstract} platelets because they are difficult to preserve and always in demand. 


\title{
Transition from standard to cGMP manufacturing in a arowina laboratorv and transolant service
}

\author{
Lawrence S. Lamb *
}

\begin{abstract}
Cellular therapeutics has become increasingly complex, moving from simple collection, volume reduction, and cryopreservation of hematopoietic cells to manufacturing of a variety of specialized cellular products for cancer immunotherapy, regenerative medicine, and cell-based gene therapies. From January 2011, the University of Alabama at Birmingham Cell Therapy Laboratory has been transitioning from a small hematopoietic processing laboratory into a fully cGMP-compliant multipurpose cell manufacturing facility. This transition has been driven by the increasing demand for advanced cell manufacturing services both within and outside of the bone marrow transplantation program and by the institution's need to offer sophisticated academic and industry based multicenter cell therapy trials to our patients. In addition to the construction of an new cell manufacturing facility, additional challenges were upgrading all core operational standard operating procedures; opening a compliance, regulatory, and quality service within the cell therapy laboratory; and addressing the changes in both the culture of our laboratory and the services that are impacted by these changes. Implementation of these changes in practice are taking place while continuing to support collection and processing for a pediatric and adult bone marrow transplant program that serves approximately 120 patients per year. These challenges are being addressed through a close partnership with our investigators, the UAB

consultants, and our principal diagnostic and manufacturing equipment suppliers. Through this collaborative effort we have incorporated new industry and $\mathrm{NIH}$-funded multicenter trials as well as initiated two new cGMP manufacturing protocols for trials unique to our institution. The first institution-specific trial will evaluate ex vivo expanded/activated haploidentical gamma/delta $\mathrm{T}$ cells in the treatment of glioblastoma multiforme. The scientific rationale for this trial has been extensively addressed in both in vitro and animal studies and in preliminary discussions with FDA and represents a simple manufacturing process ideal for the initial work of a new laboratory. The second trial will evaluate gene correction of induced pluripotent cells (iPSc) from patients with nonmalignant genetic hematopoietic diseases followed by re-differentiation of the corrected iPSc into hematopoietic progenitors for autologous transplantation. This manufacturing process is complex and has been greatly enhanced by the availability of a manufacturing system with total bio-containment suitable for extended cell manipulation and culture protocols. In summary, the challenges that exist to transitioning an existing hematopoietic processing laboratory to a cGMP manufacturing facility can be successfully addressed through partnerships with employees, academic and hospital based stakeholders, consultants, and high-value suppliers.
\end{abstract} facilities planning service, external compliance/regulatory 


\title{
Proceedings of the 2 nd Meeting of the Asian Cellular Therapy Organization
}

JSRM Code: 007030700050

ISSN $0973-7154$

\section{SANYO'S Approach to Aseptic Cell Manufacturing}

\author{
Sachihiko Kataoka*
}

Abstract

\begin{abstract}
The Biomedical Division of SANYO Electric Co., Ltd. has been engaged in the business of CPC (Cell Processing Centre = GMP grade clean room) since 2001 and we have successfully installed more than 100 facilities of these across Japan.

SANYO's objective in this area is to provide an aseptic environment to users who manipulate human cells that are not usually feasible to sterilise at the final stage. In the $\mathrm{CPC}$, it is required to design and construct a grade $\mathrm{B}$ room that is enclosed by grade $D$ or $C$ rooms because safety cabinets, $\mathrm{CO} 2$ incubators, centrifuges and cell monitoring microscope and such must be placed in a grade B room in the GMP facilities.
\end{abstract}

On the contrary, the space requirement for the traditional CPC and its running costs (electricity for air conditioning, consumables such as 2nd non-shedding gown, etc) are always issues to operate the entire CPC facilities. Due to these issues, a compactly designed yet the high performance device which offers the same level of or better aseptic environment as the CPC was gradually and increasingly hoped for by many customers.

SANYO Electric Co., Ltd has started the marketing of CPWS systems overseas at the beginning of 2011 and we successfully installed a first unit in the US this year.

In order to address these issues, SANYO introduced the unique CPWS (Cell Processing Work Station) systems in the field in 2004 and has been supplying them in Japan. The work area of CPWS system is totally closed therefore it can offer a high level of aseptic condition. Also, a $\mathrm{CO} 2$ incubator and a centrifuge can be directly connected to the CPWS system and this design makes it possible to achieve an aseptic environment in the $\mathrm{CO} 2$ incubator and the centrifuge along with the main work area. The whole area can be decontaminated by a validatable hydrogen peroxide decontamination cycle, and on top of the closed design, the CPWS system realises the high level aseptic condition.

The CPWS system which utilises the closed system conducts the air exchange only through the integrated HEPA filters therefore it eliminates the requirement of a grade $B$ room. This contributes the smaller installation space compared to the traditional CPC facilities as well as less electricity since the air conditioning system can be smaller and also 2nd gowns (consumables) are not required to wear. These benefits can support the low cost operation of facilities and this unique CPWS concept has been well accepted by many users in Japan . 


\title{
Development of Automated cell processing system R-CPX for reaenerative medicine
}

\author{
Katsumi Nakashima*
}

\begin{abstract}
It is important for the industrialization of the regenerative medicine to develop automated cell processing technologies. Especially, it is necessary not only to culture high-quality cells, but also to avoid the contamination of cells. In the research field, the skilled human technician has operated the cell processing in the clean environment. However, the human operation costs a great deal. The automated system will reduce the cost and provide high quality cells stably without contamination. We have developed the Robotized- Cell Processing eXpert system: $\mathrm{R}-\mathrm{CPX}$ using our clean robot system. In R-CPX, the operation area can be cleaned by V-PHP (Vapor-Phase Hydrogen Peroxide), and cells from plural donors can be cultivated in parallel. Using the cleaning process after the
\end{abstract}




\title{
1. The current situation of $\mathrm{CRO}$ business in Asia 2.Experience of clinical development/study in Asia. 3.Future of clinical development in Asia from CRO point of view
}

\author{
Hidemi Aida*
}

Abstract

\begin{abstract}
50 The 2nd Meeting of Senior Advisor Clinical Development Services Quintiles Transnational Japan K.K. Co-Sponsor: Quintiles Transnational Japan K.K. Hidemi Aida Clinical development in Asia, from CRO point of view Asia is rapidly becoming a new frontier for drug development as Western companies seek to develop and register their products in Asia, while emerging Asian companies seek new capabilities to globalize products. As Asia continues to grow, the need for improved healthcare will drive the need for new medicines. To fully capitalize on the R\&D opportunities presenting themselves in the growing markets of Asia, the biopharmaceutical industry will need to relinquish its faltering "go-at-it-alone" linear model of drug discovery and development in which one firm controls all the pieces, and shift towards a "wheel-andspoke" model of multiple strategic partnerships with other biopharma and service providers companies with complementary strengths and capabilities.
\end{abstract}

Asian biopharmaceutical companies face challenges that are a blend of those faced by the multinational pharmaceuticals and small biotechs. They too face rising R\&D costs, regulatory hurdles and diminishing returns, but their key challenge has been the inability to globalize their clinical development programs. An historic example is the Japanese pharmaceutical industry that has excelled in discovery, product development and commercialization within the confines of Japan, but internationally Japanese companies have few products and virtually no market share.
The past decade witnessed a sharp increase in the number of clinical trials being run in Asia. However, this increase has primarily been the "off-shoring" of patient enrollment from the West to Asia because of the cheaper and easier access to patients, while the study concept and drug development strategies remained in the US and in Western Europe. However, akin to trends in other industries, this initial phase of off-shoring of low cost, low skilled operations will soon be followed by the demand for more highly technical and knowledge-based drug development capabilities in Asia.

As part of this change it will be important to fracture the linear, monolithic "go-at-it-alone" model of biopharma -from drug discovery through development to commercialization - in favour of a model replete with multiple partnerships and strategic alliances. In the latter, companies will focus on their core strengths, and partner or outsource all other activities to other firms better at performing those tasks. This model will lend itself to the principle of "how can we work to win together?"

As part of this paradigm it will not only be important for CROs to work together with biopharma abut it will also be imperative that investigators are an integral part of the team that will deliver new and better medicines to patients faster 


\title{
Proceedings of the 2 nd Meeting of the Asian Cellular Therapy Organization
}

\section{Stem cell therapy in chronic stroke patients-Phase II trial}

\author{
Shinn-Zong Lin1,2, 3, Demeral Liu1, Woei-Cherng Shyu1, 3
}

\begin{abstract}
Our pilot clinical trial was performed in 6 chronic stroke patients (stroke duration more than 6 months) by implanting autologous hematopoietic stem cells into the infarct brain. The results show that the damaged corticospinal tract might be regenerated within 3 to 6 months after the transplantation. However, the corticospinal tract regeneration and motor improvement were much better in young stroke patients (age less than 60 years old) than the elder (age more than 61 years old). We hypothesized that the age dependent regeneration of the corticospinal tract may be related to the molecular systems of the chemoattractants and stress proteins.

The most common and strongest chemoattractant protein is the stromal-cell-derived factor-1 alpha (SDF-1 alpha) and its receptor CXCR4. These two proteins together with cellular prion protein (PrPsc) are upregulated in the acute injured brain and stem cells. Stress induced protein such as secretoneurin is also upregulated during acute brain injury. In animal studies, overexpression of these proteins not only protects the injured brain from neural apoptosis, but also attracts bone marrow as well as neural stem cell to

the injured site. Stem cells secrete various nurotrophic factors, angiogenic factors, stress proteins, and chemoattractants to enhance neuroprotection, tractogenesis and neuroplasticity. In addition, stem cells may also rejuvenate the surrounding cells.

We are currently conducting a randomized, double blind control trial for chronic stroke patients by implanting CD34 stem cells into the infarct brain. Our preliminary results show that there were a significant increment of corticospinal tract fiber density ratio (CTFDR) assessed by diffusion tensor image (DTI) in treatment group than control. The neurological improvements were better in the treatment group as compared to control patients in regarding to NIHSS, ESS, EMS, and Barthel Index. Details will be presented in the meeting.

This randomized control study demonstrates that a therapeutic strategy using G-CSF combined with autologous implantation of PBSCs mobilized by G-CSF transplantation for old stroke patients is safe, feasible, and shows preliminary evidence of improved neurological outcomes.
\end{abstract}




\title{
Proceedings of the 2 nd Meeting of the Asian Cellular Therapy Organization
}

\section{Unproven stem cell treatments: Moving targets on the global landscape}

Douglas Sipp*

\begin{abstract}
I will give a brief overview of the claims, economics, and geographical scope of the unregulated global industry (or black market) centered on the commercialization of stem cell injections for which there is no rigorous evidence of safety and/or efficacy. In particular, I will focus on recent regulatory developments and shifts in marketing tactics and regions targeted.
\end{abstract}




\title{
Proceedings of the 2 nd Meeting of the Asian Cellular Therapy Organization
}

JSRM Code: 007030700055

ISSN $0973-7154$

\section{NK cell therapy for cancer in practice}

\author{
Jun-ichi Masuyama*
}

Abstract

\begin{abstract}
Natural killer (NK) cells are a promising tool for cancer immunotherapy because of their potent killing response to a broad range of tumor cells through diverse receptorligand interactions. We present NK cell therapy for patients with cancer using a novel method for ex vivo NK cell expansion we developed at our clinic founded in 2004.

Our method induces vigorous expansion of CD3-CD56+ NK cells without using accessory cells or fetal bovine serum. Peripheral blood mononuclear lymphocytes were stimulated with GMP-regulated medical drugs in the presence of autologous plasma, and expanded in NKGM medium (Kohjin Bio) containing interleukin-2. After 14 days of culture in CO2-permeable bags $(1 \mathrm{~L} \times 2)$, the median NK cell yield was $5.9 \times 109$ cells (range; 1.6-11.0 $\times 109$ cells, $n$ $=25$ ) from $20 \mathrm{ml}$ peripheral blood of healthy donors, and its median expansion was 859-fold (range; 296- to 2700-fold) of the initial input NK cells. In vitro cytotoxicity against K562 was $60.3 \%$ (median, $n=5$ ) at an $E: T$ ratio of $1: 1$, and in vivo cytotoxicity against human pancreatic cancer cell lines was also observed in a murine model.
\end{abstract}

Although medical practice without national approval is able to be performed by medical doctor's discretion in Japan, we established the ethics committee of New City Osaki Clinic as a third party to estimate the clinical protocol for NK cell therapy. In addition to approval by the committee, patients gave written informed consent before NK cell therapy. To ensure safety and traceability of cell products, we saved all records of culture process and introduced a bar-code system to avoid cross-contamination during a GMP-based aseptic culture procedure.

We have treated 448 cancer patients until May 2011. The only adverse event was transient fever (up to $38^{\circ} \mathrm{C}$ ) in around $20 \%$ of the patients, occurring 1-2 $\mathrm{h}$ after the infusion. The survival benefit was evaluated in 33 patients with advanced pancreatic cancer who received at least 4 infusions of NK cells combined with/without chemotherapy (gemcitabine and/or S-1). The median overall survival rate was 16.0 months (range: 4-52). The 1-year survival rate was $64 \%$. Immunologic monitoring revealed a significant increase in both NK activity and the number of NK cells and NKG2D+ cells in the patients showing clinical benefit.

The described method is easy and safe, and would provide practical NK cell therapy exerting ADCC activity in antibody therapy, or cytolysis in an allogeneic setting. 


\title{
Modified donor lymphocyte infusion in the prophylaxis of relapse after HLA-mismatched/haploidentical T-cell-replete hematopoietic stem cell transplantation for advanced-stage acute leukemia: a retrospective risk factors analysis
}

Wang-Yu*, Liu Dai-Hong, Xu Lan-Ping, Liu Kai-Yan, Chen Huan, Zhang Xiao-Hui, Chen Yu-Hong, Han Wei, Huang Xiao-Jun

\begin{abstract}
The role of donor lymphocyte infusion (DLI) in the prophylaxis of relapse has not been defined. We retrospectively analyzed the data of 88 patients with advanced stage acute leukemia after HLAmismatched/haploidentical hematopoietic stem cell transplantation (HSCT) whose treatment did $(\mathrm{n}=61)$ or did not $(n=27)$ include prophylactic DLI. The two groups were compared with respect to relapse and overall survival. Further, a detailed analysis of risk factors was performed.

The 2-year cumulative incidence of relapse in patients receiving prophylactic $\mathrm{DLI}$ and not receiving prophylactic DLI were $36 \%$ and $55 \%(P=0.017)$. Estimated survival at 3

years was $31 \%$ for patients receiving prophylactic DLI and $11 \%$ for patients not receiving prophylactic $\mathrm{DLI}(\mathrm{P}=0.001)$. The three-year probability of LFS was also higher in patients receiving prophylactic DLI $(22 \%)$ than in patients not receiving prophylactic DLI $(11 \%) \quad(P=.003)$. Multivariate analysis for relapse showed that use of prophylactic DLI after transplantation was an independent prognostic factor $(p=0.025)$. Higher OS was associated with use of prophylactic DLI ( $P=0.002)$, acute myeloid leukemia $(P=0.027)$ and female sex $(P=0.023)$. Our results suggest that the prophylactic modified DLI may increase survival after HLA-mismatched/haploidentical HSCT for advanced-stage acute leukemia.
\end{abstract}




\title{
Modified donor lymphocyte infusion associated acute graft-versus- host disease after haploidentical T-cell-replete hematopoietic stem cell transplantation: incidence and risk factors
}

\author{
Chen-hua Yan*, Dai-hong Liu, Lan-ping Xu, Kai-yan Liu, Ting Zhao, Yu Wang, Huan Chen, Yu-hong Chen, \\ Wei Han, Xiao-jun Huang
}

\begin{abstract}
Objects: To investigate the profile of donor lymphocyte infusion (DLI)-associated acute graft-versus-host disease (GVHD) in haploidentical T-cellreplete hematopoietic stem cell transplantation (HSCT).

Methods: Consecutive 124 patients receiving modified DLI after haploidentical T-cell-replete HSCT were enrolled.

Results: The cumulative incidence of DLI-associated acute GVHD was $53.2 \%$ for grade II to IV and $28.4 \%$ for grade III to IV. Multivariate analysis demonstrated that the duration of GVHD prophylaxis after DLI was related to the development of DLI-associated grade III to IV acute GVHD $(P<0.05)$. The cumulative incidence of grade III to IV acute GVHD in patients with prophylaxis more than 6 weeks, 4 6 weeks, $2-4$ weeks and less than 2 weeks were $9.3 \%$,

$14.4 \%, 31.6 \%$ and $49.5 \%$, respectively ( $P=0.018)$. Besides, DLI-associated grade III to IV acute GVHD was the only risk factor for overall survival $(P=0.038, O R=2.869)$ and transplant-related mortality $(P=0.018, O R=3.296)$, but not risk factor for relapse after $\mathrm{DLI}(\mathrm{P}=0.840)$.

Conclusion: This was the first study to investigate the incidence and risk factors of DLI-associated acute GVHD in haploidentical T-cell-replete HSCT, and for the first time confirmed that the duration of GVHD prophylaxis after DLI was the only risk factor for the development of grade III to IV acute GVHD. DLI with GVHD prophylaxis more than 4 weeks was associated with a lower incidence of grade ? to ? acute GVHD. Besides, this study also showed that DLIassociated grade III to IV acute GVHD was the only risk factor for inferior OS and higher TRM.
\end{abstract}




\title{
Lymphoid commitment in human bone marrow CD34-positive cells reflects aging of hematopoietic stem cells.
}

\author{
Yuichi Kodama*, Azumi Miyazono, Yasuhiro Okamoto, Yuni Yamaki, Koichiro Kurauchi, Takuro Nishikawa, \\ Takayuki Tanabe, Yuichi Shinkoda, Yoshifumi Kawano
}

\begin{abstract}
(Background) In the clinical setting, pediatric patients with hematopoietic disorders often undergo hematopoietic stem cell transplantation (HSCT) from adult donors. However, it is not yet clear whether hematopoietic stem cells (HSCs) from adult donors have enough potential to maintain hematopoiesis throughout the life of pediatric recipients after HSCT. Do transplanted HSCs have the same longevity as the originals? In a mouse model, a clonal analysis of repopulating HSCs demonstrated that lymphoidbiased HSCs are lost and myeloid-biased HSCs accumulate with aging. As a first step in understanding the aging of HSCs in humans, we analyzed the changes in commitment of human CD34-positive cells based on the quantity of genetic lymphoid/myeloid markers.

(Material and Methods) Bone marrow cells in 35 subjects were included in this study. The cells were harvested from healthy donors for HSCT and pediatric patients without hematological disorders. CD34-positive cells were isolated

according to the manufacturer's protocol. After cDNA was synthesized by CD34-positive cells, mRNA expression levels of ikzf1 and cebpa, which were used as the target genes of lymphoid and myeloid markers, respectively, were measured by real-time PCR. The data for each sample were normalized relative to the expression level of GAPDH and calculated by the 2-?Ct method.

(Results) The median age of the donors was $21 \mathrm{y}$ (range $0.2-44 \mathrm{y}$ ). The purity of CD34-positive cells was greater than $90 \%$. Both target genes were quantitatively detected in all samples. There was an inverse correlation between the expression level of ikzf1 and the donor's age $(r=-$ $0.358, p=0.032$ ). In contrast, there was no correlation between the expression level of cebpa and the donor's age.

(Discussion) The mRNA level of ikzf1 in purified CD34positive cells may reflect the age of HSCs in human bone marrow.
\end{abstract} with a CD34 MicroBead kit and autoMACS (Miltenyi Biotec) 


\title{
The Study on the Source of Engraftment of Mesenchymal Stem Cells in Patients after Different Source of Hematopoietic Stem Cell Transplantation
}

\author{
Jing Wang, Xiao-Jun Huang, Yu-Hong Chen, Lan-ping Xu, Dai-hong Liu, Huan Chen, Rui-Qin Hou, Xiao-Ying
} Qin, Kai-yan Liu*C

\begin{abstract}
Objective: To study the engraftment source of mesenchymal stem cells of bone marrow origin in patients after different source of hematopoietic stem cell transplantation.

Method: In this study, hematopoietic stem cells from 32 clinical donors of different sex, resulting in successful engraftments, were selected for transplantation and classified into three groups for research. In which, 14 cases in group $A$ with bone marrow plus recruited peripheral hematopoietic stem cell transplantation, group B contained 8 cases of simultaneous re-transfusion of mesenchymal stem cells from the second donor and group $C$ had 10 cases of simultaneous re-transfusion of umbilical blood from the second donor. Bone marrows from 32 patients with successful engraftments of hematopoietic transplantation were selected and sub-cultured with mesenchymal stem cells. Flow cytometry (FCM) was used to measure the expressive characteristics of surface antigens in MSCs. Denaturing high performance liquid

chromatography (DHPLC) in combination of polymerase chain reaction for amplification of short tandem repeats (STR-PCR) was used to measured the engraftment statuses of MSCs of the fifth generation in patients. Fluorescence in situ hybridization (FISH) proved the sex origins of MSCs of the fifth generation in 32 patients.

Results: In 32 cases, progenies of up to the fifth generation were successfully cultured. The results of FCM demonstrated that the expressions of CD14+ and CD45+ cells were lower than $0.04 \%$ in MSCs of the fifth generation. DHPLC and FISH had similar results. One patient from the group with simultaneous re-transfusion of mesenchymal stem cells from the second donor had temporary transplantation of MSCs from the donor as well. The MSCs in remaining 31 patients all originated from the patients themselves. Conclusion: After transplantation, MSCs in patient are still from host origin. In patient with transplantation of MSCs from second donor, phenomenon of temporary chimerization of MSCs was seen.
\end{abstract}




\title{
Novel natural killer (NK) -cell expansion system for producing large amount (>109 cells) and high purity (>90\%) of CD3+ CD56+ cells
}

\author{
Mitsuko Ideno1*, Mie Yabuuchi1, Meiko Jin1, Tatsuji Enoki1, Naoyuki Sakamoto2, Takeshi Ishikawa3, Satoshi \\ Kokura3, Junichi Mineno1, Mitsuko Ideno, Meiko Jin, Tatsuji Enoki, Junichi Mineno
}

\begin{abstract}
Purpose and background: NK cell-based adoptive immunotherapy is one of expecting approaches against any advanced cancers. However, there are few convenient and efficient method to provide a large amount of functional NK cells having a high proportion of CD3-CD56+ cells. In this study, we show a novel NK-cell expansion method using OK432 (Picibanil), IL-2 and RetroNectin® induced T cells $(\mathrm{RN}-\mathrm{T})$ as a stimulator.

Methods: RN-T were prepared by previously reported method in advance, and processed to use as stimulator cells. NK cells were expanded from PBMCs by stimulating with modified RN-T, OK-432 and IL-2, then cultured for 1622 days. We also established large-scale culture system using gas-permeable culture bag, CultiLife®215 and CultiLife $®$ Eva, for future clinical application.

Results: After 16-18 days culture, the total cell expansion rate reached more than 200 -fold and the final cell population contained over $90 \%$ of CD3-CD56+-NK cells.

Furthermore, almost all expanded cells displayed functional cell surface molecules such as NKG2D and CD16 implicated in cytotoxicity and antigen dependent cell cytotoxicity (ADCC). Indeed, the expanded NK cells showed strong cytotoxicity against various tumor cell lines such as leukemia, lung, breast, melanoma, and rectal, and also ADCC in combination with tumor antigen specific antibody in vitro. In largescale culture system, we could obtain $109 \sim 1010$ cells containing a high proportion (>90\%) of CD3-CD56+-NK cells from $50 \mathrm{~mL}$ of peripheral blood. Next, we tested for the ability of this system to expand NK cells from advanced cancer patients' blood. As a result, the mean of total cell expansion rate after 21-22 days culture was 669-fold (range $191 \sim 1710: n=5$ ) and more than $94 \%$ of CD3-CD56+-NK cells were contained in all cases.

Conclusion: This innovative ex vivo NK-cell expansion system provides convenient tool for obtaining large amount and high purity of CD3-CD56+ cells.
\end{abstract}




\title{
Development of a novel stimulation method to expand CIK cells containing high proportion of CD3+ CD56+ cells
}

\author{
Mitsuko Ideno*, Meiko Jin, Tatsuji Enoki, Junichi Mineno
}

Abstract

\begin{abstract}
Background: Cytokine-induced killer (CIK) cells are highly efficient cytotoxic effector cells generated by culturing PBMCs in the presence of anti- CD3 mAb, IL-2 and IFN-?, and they characteristically contain the "NK-like $\mathrm{T}$ cell" population expressing both $\mathrm{T}$ cell marker CD3 and NK cell marker CD56 (CD3+CD56+). Among CIK cells, NK-like T cells $(\mathrm{CD} 3+\mathrm{CD} 56+)$ are the main effector cells and demonstrate the most potent cytolytic activity to various types of tumor cells. Therefore, it is suggested that the technology for increasing the NK-like T cells proportion in CIK cells is a key to success for enhancing the clinical effects. We have previously reported the efficient T-cell expansion method using immobilized anti-CD3mAb and RetroNectin ${ }^{\circledR}(\mathrm{RN})$. In this method, NK-like $\mathrm{T}$ cells are not so much in expanded $T$ cells even though a large amount of naive $T$ cells can be obtained. Here, we show a novel expansion method for producing CIK cells by two step stimulations, with initial anti-CD3mAb/RN and second "supplement X".
\end{abstract}

Results: By combining anti-CD3mAb/RN (1st) and supplement X (2nd) stimulations, PBMCs proliferated 250700 fold on day14-15, and the expanded cells contained higher proportion (40-60\%) of CD3+CD56+ than ones proliferated without second stimulation by "supplement X"

(10-20\%). Most of the expanded cells expressed both CD8 and NKG2D, and showed strong cytotoxicity and IFN? release. Next, we evaluated which population of CD3+CD56+ or CD3+CD56- cells showed strong cytotoxicity. As a result, CD3+CD56+ was more potent than CD3+CD56- population. Further, we established largescale culture system using gas-permeable culture bags, CultiLife ${ }^{\circledR} 215$ and CultiLife $\AA$ Eva. With this system, we could obtain a large number of CIK cells $(>1010)$ containing high proportion of NK-like T cells (CD3+CD56+) ( ? 50\%) from $50 \mathrm{~mL}$ of blood.

Conclusion: We expect that this newly devised method for CIK expansion would be an effective tool for CIK-based immunotherapy. 


\title{
Depletion of CD3-positive cells enables to generate purified and expanded human natural killer cells from peripheral blood mononuclear cells showing strong killer activity
}

\author{
Satoru Saito*, Yui Harada, Yoshikazu Yonemitsu
}

\begin{abstract}
PURPOSE: Natural killer (NK) cells play a crucial role of the innate immune system, and clinical studies to treat advanced malignancies using NK cells are emerging worldwide; however, overall purity and numbers of NK cells and their antitumor potencies are unsatisfactory as to obtain clinical outcome. We therefore attempted to establish an efficient method to purify and expand the highly activated NK cells ex vivo.

METHODS: Under approval of the institutional ethical committee, peripheral blood mononuclear cells (PBMC) were collected from healthy volunteers and patients with advanced malignancies. CD3+-cells were depleted by magnetic beads, and CD3-depleted PBMC (CD3-PBMC) could be expanded in the presence of high concentration hIL-2 and 5\% human autoserum for 14days to expand NK cells. The characteristics of these CD3-/CD56+ NK cells were subjected to the expression of surface markers, CD107a mobilization and cell-mediated cytotoxicity against K562 cells in vitro compared with those of primary NK cells.

RESULTS: CD3-PBMC could be expanded in the presence of high concentration hIL-2 and the majority of the expanded cells were CD3-/ CD56+ (>90\%) after 14days culture. The expanded NK cells expressed various killercell immunoglobulin-like receptors (KIRs), natural cytotoxicity receptors (NCRs), CD69, NKG2D, and CD16. CD107a mobilization assay and cytotoxicity assay revealed that expanded NK cells could kill K562 target cells more efficiently than that seen by primary NK cells.

CONCLUSION: We have established new methods to deal with the current issues underlying NK-based immunotherapy. Overall purity of expanded NK cells was sufficiently expressing various receptors, including KIRs and NCRs. Moreover, these cells showed strong cytotoxic activity against K562; and importantly, we could obtain these NK cells from PBMCs from patients with advanced malignancies using this method. Therefore, this novel method may contribute to provide a new tool for clinical cancer immunotherapy.
\end{abstract}




\title{
Proceedings of the 2 nd Meeting of the Asian Cellular Therapy Organization
}

\section{Effect of Herceptin is increased by ex vivo cultured NK cell via antibody-dependent cellular cytotoxicity (ADCC).}

Takako Wakeda1*, Sul Hee Yoon2, Yoshinori Ikarashi3, Kazunori Aoki3, Tsukasa Shinohara1, Yuni Yamaki1, Yuji Heike1

\begin{abstract}
Herceptin is a widely used antibody-drug for current breast cancer treatment. However, a number of patients respond poorly to Herceptin, making the development of a new therapy that enhances the effect of Herceptin necessary. The antitumor effect of Herceptin arises from the antibodydependent cellular cytotoxity (ADCC) of Natural Killer (NK) cell. Hence a therapy that combines NK cell activity with Herceptin is a promising approach, and it is this possibility that we have examined in this study. First, a method for expansion of NK cells ex vivo was established, and then the expanded cells were evaluated for Herceptinmediated ADCC against the breast cancer cell lines. In our experiment, the NK cells were cultured from CD3-negative cells obtained from peripheral blood mononuclear cells by magnetic bead selection using a newly-developed culture medium and closed bag system. The fresh cells on day0 and the expanded cells were evaluated for surface

markers, intracellular cytokine production and Herceptinmediated ADCC against the breast cancer cell lines MCF-7 and MDA-MB-231. After expansion for 14-23 days, the percentage of CD56+ cells was higher than $99 \%$ and the CD3+ cells stayed below $0.1 \%$, and the pure population of NK (CD3-/CD56+) cells increased to $31.9 \pm 12.0$ fold. Expression of NK activation markers NKG2D, CD107a, Perforin, and GranzymeB were extremely high before and after expansion. The cultured NK cells showed a high level of cytotoxity against the breast cancer cell lines and an even higher level in the presence of Herceptin. In conclusion, a novel NK cell expansion method was established and the expanded NK cells showed a high level of Herceptin-mediated ADCC. These findings suggest that NK cell therapy combined with Herceptin would be a novel clinical immunotherapy for breast cancer patients with a poor response to Herceptin.
\end{abstract}




\title{
Synergistic anti-cancer effect of large scale simultaneous expanded NK and $y \delta T$ cells with monoclonal antibody drugs
}

\author{
Xuewen Deng ${ }^{*}$, Mie Nieda, Hiroshi Terunuma
}

\begin{abstract}
Aims: ADCC is an important mechanism of therapeutic monoclonal antibody drugs (mAbs) for anti-cancer. Natural killer (NK) cells and $ү \delta T$ cells are major effectors of ADCC through Fc receptor (CD16). We aim to develop a new method with simultaneous expansion of NK cells and $ү \delta T$ cells (NK/ $\mathrm{Y} \delta \mathrm{T})$ for the purpose of combination immunotherapy with mAbs.

Methods: NK/ $\mathrm{\delta T}$ were simultaneously cultivated from PBMCs in the presence of OK432, zoledronate and IL-2 with immobilized anti-CD16 antibody without any feeder layers.

healthy donors $(n=3)$ for 3 weeks were 3425 times and 2256 times, respectively. A large number of CD16+ cells were obtained. CD16 expressed in most of the NK cells and a part of $y \delta T$ cells. In particular, the percentage of CD16+ $y \delta T$ cells from the new method is higher than that from a conventional expansion method using zoledronate. $\mathrm{NK} / \mathrm{\gamma} \delta \mathrm{T}$ had high cytotoxicity against CD20+ cell lines and induced the ADCC by Rituxmab.

Conclusion: We simultaneously expanded NK/ yסT possessing high ADCC effects in vitro. These cells may be useful immune cells for a combination immunotherapy with mAbs
\end{abstract}

Results: Median of expansion rate of NK/ үסT cultured from 


\title{
Proceedings of the 2 nd Meeting of the Asian Cellular Therapy Organization
}

\section{Requirements for common chain family cytokines for adoptively transferred $y \delta \mathrm{T}$ cell function and homeostasis in vivo}

\author{
Kazuhiro Kakimi1*, Takamichi Izumi1, Makoto Kondo1, Nao Fujieda1, Atsushi Kondo1, Naohisa Tamura1, \\ Ryuji Maekawa2, Hirokazu Matsushita1
}

\begin{abstract}
We have conducted clinical trials of adoptive $\gamma \delta T$ cell therapy for the treatment of cancer. Peripheral blood mononuclear cells (PBMC) were harvested by apheresis and $ү \delta T$ cells had been expanded ex vivo for 14 days by culture with zoledronate $(5 \mu \mathrm{M})$ and $\mathrm{IL}-2(1000 \mathrm{IU} / \mathrm{ml})$. Patients received $>1 \times 109$ y $\delta T$ cells every 2 weeks. Infused Y $\delta T$ cells gradually accumulated in patients' peripheral blood and accounted for $10 \%$ of PBMC as late as 3 months after the final injection, even without IL-2 administration in vivo. These cells maintained the ability to release cytotoxic granules (detected by CD107 assay) and produce IFN-y in response to re-stimulation. To determine the factors contributing to $y \delta \mathrm{T}$ cell survival in vivo, we investigated

family cytokines at the time of infusion. IL-2Rß and $y c$, but not IL-7R $\alpha$, IL-15R $\alpha$ or IL-21R, were present on these cells, and although the IL-2Ra was upregulated at the initiation of culture, it was only very weakly expressed at the time of transfer. When $\gamma \delta \mathrm{T}$ cells were maintained for longer periods in culture, IL-15 but not low dose IL-2 and IL-7 supported their survival. Because IL-15 can be transpresented with IL-15Ra, y $\delta \mathrm{T}$ cell expressing IL-2Rß and $y c$ can respond more efficiently to IL-15 than IL-2. These results suggest that tissue-specific yc-dependent signals in response to IL-15 might affect the survival and function of infused $\gamma \delta T$ cells, and hence materially influence the success of immunotherapy.
\end{abstract} their expression of receptors for common $y$ chain $(\gamma c)$ 


\title{
Proceedings of the 2 nd Meeting of the Asian Cellular Therapy Organization
}

\section{Immune tolerance induction to FVIII in hemophilia A using autologous endothelial progenitors}

\author{
Hideto Matsui1*, David Lillicrap2, Mitsuhiko Sugimoto1, Midori Shima1
}

\begin{abstract}
Currently, the development of FVIII antibodies (FVIII inhibitors) remains the most significant treatment complication for hemophilia A patients. These inhibitory antibodies usually target functional epitopes on FVIII, thus rendering FVIII replacement therapy ineffective. We have used the hemophilia $A$ mouse model to evaluate a novel strategy for the induction of reduced immunological responsiveness to FVIII. With a view to establishing immune tolerance to the FVIII, autologous blood outgrowth endothelial cells (BOECs) transduced with a lentiviral vector encoding canine FVIII (cFVIII) were mixed with Matrigel and injected subcutaneously (2 x106 cells/injection) into hemophilia A mice that had previously been infused repeatedly with cFVIII in order to develop a robust anti-cFVIII inhibitors (25-45 BUs). The levels of inhibitors began to decline within 6 weeks of BOEC

transduced BOECs the inhibitor levels in all mice had declined to < 5BUs. When these partially tolerized mice were re-challenged with the intravenous injection of cFVIII, they showed a rapid increase of FVIII inhibitor titers. In contrast, partially tolerized mice that were re-challenged with subcutaneous cFVIII showed no increase in inhibitor titers. Increased numbers of CD4+CD25+Foxp3+ regulatory $T$ cells were detected in the spleen (4\%) and subcutaneous draining lymph nodes $(7 \%)$ of the partially tolerized mice and adoptive transfer of CD4+ splenocytes and draining node lymphocytes from these animals to naïve hemophilic mice transferred tolerance to cFVIII. These results indicate that genetically-modified BOECs implanted subcutaneously in a Matrigel scaffold may provide a novel immunemodulating therapy for elimination of FVIII inhibitors in hemophilia A patients.
\end{abstract} implantation in all mice and after 2 or 3 implantations of 


\title{
Proceedings of the 2 nd Meeting of the Asian Cellular Therapy Organization
}

\section{An Evaluation of CD34+ Cells in the Bone Marrow of various age groups of Indian Population}

\author{
Abraham S1, 2*, Manjunath S1, Senthilkumar R1, Murugan P1, Thamaraikannan P1, Srinivasan T1, Preethy \\ S1,3, Dedeepiya V1
}

\begin{abstract}
Background: Transplantation of bone marrow Hematopoietic Stem cells are indispensable to replace the blood forming system in hematological malignancies. The engraftment after transplantation depends on total CD34+ count. We report a comparative analysis of the of CD34+ cells from bone marrow of people of different age groups from within India.

Materials and Methods: Patients enrolled for autologous bone marrow mononuclear cell injection for spinal cord injury with normal vital parameters were included in this study. The Bone Marrow (BM) tapped from the posterior iliac crest preserved under 10 deg Celsius was transported to a central lab within 12 hours of harvesting. The Mononuclear cells (MNC) were isolated using density fractionation method and the CD34+ cells were analyzed using flow cytometry. The isolation of MNCs and flow cytometry for all the samples were done in a single

Results: The total number of samples evaluated was 332 and the CD34+ Cell percentage in the various age groups were $1.0569 \%$, in those belonging to the age group of $0-20$ years it was $1.04 \%$ in the $21-40$ year group, $0.96 \%$ in the $41-60$ year group and $0.93 \%$ in the $61-80$ year group. The difference in CD34+ cells percentage across these five groups showed a decreasing trend but didn't reach a statistical significance.

Conclusion: Our results imply that the percentage of CD34+ cells decrease with increasing age. This trend needs further analysis in a larger population. With the recent literatures suggesting Colony Forming Units (CFU) as a strong independent predictor of engraftment in cord blood or bone marrow transplantation, we are extending this study to assess the CFU in bone marrow sam ples of various age groups which may reveal further relevance of their engraftment capability.
\end{abstract} laboratory set-up following all GMP and GLP protocols. 


\title{
Sipuleucel-T: Adaptive Immune Response and Product Characterization from the Phase III IMPACT Trial
}

\author{
Nadeem Sheikh ${ }^{1}$, Daniel Petrylak ${ }^{2}$, Christian Poehlein $^{1}$, James Whitmore $^{1}$, Frances Stewart $^{1}$, Corazon dela Rosa $^{1}$, \\ David Urdal ${ }^{1}$
}

\begin{abstract}
Introduction: Sipuleucel-T is an autologous cellular immunotherapy designed to stimulate an immune response to prostate cancer and is FDA-approved for the treatment of asymptomatic or minimally symptomatic metastatic castrate resistant (hormone refractory) prostate cancer (mCRPC). Sipuleucel-T is manufactured from peripheral blood mononuclear cells (PBMCs) isolated from fresh leukapheresis at weeks (wks) 0, 2, and 4. PBMCs are cultured ex vivo with a recombinant fusion protein, PA2024 (composed of prostatic acid phosphatase [PAP] and granulocyte-macrophage colony-stimulating factor [GMCSF]). Control patients (pts) received a product made without PA2024 culture. In the Phase III IMPACT trial, pts who received sipuleucel-T had a 4.1 months improvement in median survival $(\mathrm{HR}=0.78 ; 95 \%$ confidence interval: $0.61,0.98 ; \mathrm{P}=0.03$ ) compared to pts who received control product. Here, we assessed the immune response profiles generated by sipuleucel-T and correlate product parameters and post-treatment immune responses with overall survival (OS).

Methods: Prior to infusion in pts, all lots of sipuleucel-T and control product were evaluated for antigen presenting cell (APC) activation, as assessed by the upregulation of CD54. In addition, large CD54+ cell content and the total nucleated cell (TNC) count were also determined. Culture supernatants from sipuleucel-T or the control product were assayed for cytokine production (IL-2, TNFa, and IFNY), while cellular immune responses ( $T$ cell proliferation and IFNy ELISPOT) were assessed against the antigens (PA2024 and PAP) in pre-culture cells, in a subset of pts, at wks 2 and 4. Antibody responses were measured by ELISA to the antigens. Post-treatment cellular and humoral immune responses were assayed at wks 6, 14, and 26.

Results: APC activation was evident in sipuleucel-T, but not

in control pts. The magnitude of APC activation in sipuleucel-T pts was greater at wk $2(P<0.001)$ and wk 4 $(P<0.001)$ compared to wk 0 . Cellular immune responses to

PA2024 and PAP were not evident in the sipuleucel-T pts at wk 0 , but were detected at wk 2 and increased at wk 4 , whereas control pts had no responses against either antigen. Cytokines were not present in control cultures, but were present in sipuleucel-T cultures at wks 2 and 4 only. Post-treatment proliferative responses to both antigens increased from pre-treatment in sipuleucel-T pts only and were maintained through wk $26(\mathrm{P}<0.001)$. Post-treatment IFNy ELISPOT responses to both antigens were also present in sipuleucel-T pts only and were maintained through wk $26 \quad(\mathrm{P}<0.001$ compared to pre-treatment), demonstrating a memory $\mathrm{T}$ cell response. Antibody responses were only evident in sipuleucel-T pts after treatment and were maintained up to wk $26(\mathrm{P}<0.001)$. Both antibody responses seroconverted to $\lg G$, indicating a memory $B$ cell phenotype. Each lot release parameter of sipuleucel-T correlated with OS: CD54 upregulation $(P=0.123)$, cumulative CD54+ cell count $(P=0.016)$, and TNC count $(P=0.008)$. In addition, there was evidence of correlations between OS and cellular responses (proliferation at week $14[\mathrm{P}=0.057]$ and IFNy ELISPOT at week $26[P=0.049])$, and humoral response at week 6 $(\mathrm{P}=0.079)$.

Conclusions: Sipuleucel-T is a product that is designed to elicit an antigen-specific memory $\mathrm{T}$ cell response and a long-lasting humoral response. These responses imply the presence of functional antigen-specific $T$ cells generated as consequence of priming by the first infusion, and boosting by the second and third infusions. There is evidence of correlations between product parameters and OS as well as immune responses and OS.
\end{abstract}




\title{
Proceedings of the 2 nd Meeting of the Asian Cellular Therapy Organization
}

\section{Cytokine-based log-scale expansion of functional human dendritic cells from PBMC}

\author{
Yui Harada*, Satoru Saito, Yoshikazu Yonemitsu
}

\begin{abstract}
[PURPOSE]: Dendritic cells (DCs) play a crucial role in maintaining the immune system. Though DC-based cancer immunotherapy has been suggested to hold potential to treat various malignancies, clinical efficacies are still insufficient in many human trials. We proved that this antitumor effect depends on the number of DCs (Kato T., et al. Neoplasia 2010), and it is necessary to prepare an enough number of DCs for effective treatments of tumors. In this study, therefore, we attempted to expand functional human DCs ex vivo with new technologies.

[Materials and Methods]: Peripheral blood mononuclear cells (PBMCs) were obtained from healthy volunteers and cancer patients. CD3-depleted PBMCs were expanded and differentiated into DCs in the presence of cytokine cocktails for several weeks by floating cultivation. Expanded DC properties were analyzed, and compared with those of conventional DC.

[RESULTS]: Total cells increased approximately $10-100$

fold after 5 weeks culture and $>80 \%$ of expanded cells expressed CD11c. Thus, by this method, $10-100$ times more CD11c+ cells could be obtained than conventional procedures could. As are seen in conventional DCs, expanded DCs showed dendrites after maturation, and endocytotic activities. Expanded DCs also expressed HLA$\mathrm{DR}$, adhesion molecules, and co-stimulatory molecules and produced inflammatory cytokines as well as conventional DCs did. Functionally, MLR assay revealed that expanded DCs could stimulate allogenic T-cell proliferation to the same extent as conventional DCs.

[CONCLUSIONS]: We established a new culture method to expand human DCs. Expanded DC had properties that were required to obtain therapeutic gain. We expect that this technology will be able to contribute largely to both basic and clinical research of human cancer immunotherapy. DC expansion technology will improve therapeutic gain of cancer and alleviate patients' burden of apheresis.
\end{abstract}




\title{
Proceedings of the 2 nd Meeting of the Asian Cellular Therapy Organization
}

\section{Efficient antigen presentation by DCs loaded with antigens by electroporation}

\author{
Koji Nagaoka1*, Masashi Takahara1, Ryuji Maekawa1, Takayuki Yoshimoto2, Tsuyoshi Teruya3, Hiroshi \\ Shiroma3
}

\begin{abstract}
For successful dendritic cell (DC) therapy of cancer, it is important to choose the optimal method to load DCs with antigens to induce strong antitumor immunity, particularly tumor-specific CTL responses. In this study, we performed side-by-side comparisons of two antigen loading methods, coincubation (Co) and electroporation (Ep), using murine bone marrow DCs. First we examined the uptake of FITCdextran, and showed more efficient uptake of FITC-dextran by DCs loaded by Ep than those by Co. Next, to compare the efficiency to prime CD4 and CD8 T cells, DCs were loaded with OVA by Co or Ep, and then cocultured with OVA-specific TCR-transgenic OT-I CD8 T cells or OT-II

and cytokine secretion of OT-II T cells, but had little effect on OT-I T cells. In contrast, OVA-Ep-DCs potently stimulated both OT-I and OT-II T cells to expand and secrete cytokines, such as IL-2 and IFN-?. Using monoclonal antibody specific for MHC classl/OVA peptide (257-264) complex, we confirmed efficient presentation of the OVA peptide on MHC class I by OVA-Ep-DCs. These results suggest that DCs loaded with tumor antigens by Ep would have greater abilities to induce anti-tumor immunity including CTL responses than those by Co. Now we are comparing in vivo anti-tumor effects of DCs loaded with tumor antigens by these two methods.
\end{abstract} CD4 T cells. OVA-Co-DCs induced moderate expansion 


\title{
Proceedings of the 2 nd Meeting of the Asian Cellular Therapy Organization
}

\section{Tissue Factor-triggered Procoagulant Activity of Murine/Human Mesenchymal Stem Cells}

\author{
Kohei Tatsumi1*, Kazuo Ohashi1, Yoshinori Matsubara2, Ayako Kohori2, Hiroshi Kakidachi2, Akihiro Horii2, \\ Takanori Iwata1, Teruo Okano1
}

Abstract

\begin{abstract}
[Background]Mesenchymal stem cells (MSCs) have been highlighted in the field of regenerative medicine for their availability and multipotency. However, it has been reported that MSC may associate with cell-infusion-related events including thromboembolism after being administered intravenously. This study investigated the procoagulant property of MSCs.

[Methods]As a preliminary study, we administered $1.5 \mathrm{x}$ 105 cells of murine adipose-derived MSC (ASCs) to wildtype mice via tail vein, and found that 11 out of 13 mice died within $24 \mathrm{~h}$ after injection. This fatal event was found to be associated with thromboembolism in the lung, heart, or liver. In order to investigate precise mechanism of this ASC-related event, we assessed ASCs-derived procoagulant properties with (1) citrated whole-blood using ROTEM and (2) citrated plasma using a coagulometer. We also performed the immunostaining and gene expression analysis of ASCs for mouse tissue factor (TF), and a clotting assay using normal plasma (NP) and factor VIIdeficient plasma (F7DP).
\end{abstract}

[Results]ROTEM analysis demonstrated significant shortenings of CT and CFT, and increased a-angle in ASCs samples. Plasma clotting assay also showed the procoagulant property of ASCs in a cell-number dependent manner. We also found that ASCs expressed high TF mRNA, and TF was strongly expressed around the cell surface. ASCs also shortened the clotting time of NP, while the time of F7DP was unable to be shortened as clearly as that of NP. Further investigations were conducted using human ASCs and found that human ASCs also possess strong procoagulant activity triggered by TF.

[Conclusions]ASCs express TF around the cells, and TF may trigger the activation of extrinsic coagulation pathway, leading unexpected thromboembolism. An optimal inhibition for TF by some anti-coagulant agents is recommended for securing the clinical safety of systemic ASCs administration. 


\title{
HLA-G Molecules Are Important In Alleviation Of Acute Lung Injury By Placenta Chorion Mesenchymal Stem Cells (pcMSCs) Therapy In Mice Model.
}

\author{
Thai-Yen Ling1*, Meng-Shiue Wu1, Yi-Ting Hsieh1, Jo-Chi Hsu1, Hung-Chih Lai2, Fu-Chuo Peng3, Jie-Yang \\ Jhuang4, Yao-Chang Chen5
}

\begin{abstract}
Placenta derived mesenchymal stem cells (PDMCs) had been demonstrated by using different protocols. Recently, we newly developed a novel type of MSCs from the chorionic membrane of the human term placenta, named pcMSCs.

In comparison of pcMSC and PDMCs, pcMSCs are distinguished with its unique resources of chorionic layers, rather than the whole, unseparted placenta of PDMCs. RT$P C R$ results indicate pcMSCs express various isoforms of HLA-G, including membrane forms (HLA-G1, -G2, -G3, G4) and soluble forms (HLA-G5, and -G6). Moreover, immunohistochemistry (IHC) and immunofluorescence (IFC) showed that HLA-Gs was strongly expressed only on the chorionic membrane. It was feasible to speculate that pcMSCs may secret more HLA-Gs than PDMCs.
\end{abstract}

Abstract

In our preliminary studies, pcMSCs were used as therapeutic cells to treat paraquat induced acute lung injury in animal models. After the therapy, the results showed that at day- 6 , the survival rates of mice increased from $8 \%$ to
$35 \%$ and decreased the infiltration of neutrophil into lung tissues and alveolar spaces; however, other therapies, including treatment with dexamethasone and other cell types did not increase the survival rates. These results indicated that pcMSC cell therapy significantly restored lung function.

Since HLA-G had been documented as potential immunoregulary molecules, we hypothesized that pcMSCs may decrease neutrophils infiltration in lung tissues through the action of HLA-G.s. This interaction between HLA-G and HL60 cells were examined using a co-culture migration system. It showed pcMSCs down-regulated the migration of differentiated neutrophil-like HL-60 cells, while administration of HLA-G neutralizing antibody blocked the down-regulation. Hence, it was speculated that HLA-G was the main molecule to inhibit the migration of neutrophil.

The results suggest that HLA-G molecules were important in alleviation of acute lung injury by pcMSC therapy in mice model. 


\title{
Proceedings of the 2 nd Meeting of the Asian Cellular Therapy Organization
}

\section{Useful cell lines (PMDC05/11) of plasmacytoid dendritic cell for cellular immunotherapy}

\author{
Masuhiro Takahashi1*, Miwako Narita1, Akie Yamahira1, Norihiro Watanabe1, Shigeo Hashimoto2, Yoji Yuki3
}

\begin{abstract}
Although cellular immunotherapy using antigen-specific CTLs against tumors and severe infections is a promising strategy, one of the problems is hardship in constant supply of efficinet antigen presenting cells for generating such CTLs. We established a plasmacytoid dendritic cell (pDC) line (PMDC05) from leukemia cells of pDC leukemia. PMDC05 cells were positive for CD4, CD56, CD33, HLADR, CD123 (IL-3Ra) and CD86 in the absence of lineage markers. mRNA of TLR1, TLR2, TLR4, TLR7 and TLR9 were clearly expressed with the prominence of TLR7 expression. Production of IFN-a and IL-12 in PMDC05 was enhanced by stimulation with CpG-A and LPS, respectively. PMDC05 cells were separated into two fractions according to the expression of BDCA1 and CD123. BDCA1-CD123+ cells were found to be pDC-like cells by their morphology, surface phenotypes, TLR expression and IFN-a production. BDCA1-CD123+ cells were demonstrated to have a proliferating capacity and revealed the ability to transform to BDCA1+CD123- cells

demonstrated the possibility of transformation from pDCs to mDCs in human DC lineage. In addition, we investigated the applicability of PMDC05 for cellular immunotherapy. By stimulation with LPS, PMDC05 showed enhancement in the expression of antigen presentation-associated surface molecules and production of cytokines (IL-12p70 and TNFa). The antigen presenting ability was markedly increased in PMDC05 stimulated with LPS. By co-culturing of CD8+ T cells with LPS-stimulated and WT1 or CMVpp65 peptidepulsed PMDC05, WT1 or CMVpp65 tetramer+ cytotoxic T lymphocytes were efficiently generated. PMDC11 cells, which were established by transfecting PMDC05 with CD80 gene by retrovirus-vector, showed an enhanced expression of HLADR as well as CD80 and a remarkable antigen presenting ability to allogeneic lymphocytes with an increased stimulation on IFN-? production by the lymphocytes. These findings reveal the applicability of PMDC05 and PMDC11 in cellular immunotherapy for tumors and severe infections.
\end{abstract} which showed $\mathrm{mDC}$-like properties. These data 